\title{
A consistency improving method in the analytic hierarchy process based on directed circuit analysis
}

\author{
WU Shihui", LIU Xiaodong, LI Zhengxin, and ZHOU Yu \\ Equipment Management and UAV Engineering College, Air force Engineering University, Xi' an 710051, China
}

\begin{abstract}
Test of consistency is critical for the analytic hierarchy process (AHP) methodology. When a pairwise comparison matrix (PCM) fails the consistency test, the decision maker (DM) needs to make revisions. The state of the art focuses on changing a single entry or creating a new matrix based on the original inconsistent matrix so that the modified matrix can satisfy the consistency requirement. However, we have noticed that the reason that causes inconsistency is not only numerical inconsistency, but also logical inconsistency, which may play a more important role in the whole inconsistency. Therefore, to realize satisfactory consistency, first of all, we should change some entries that form a directed circuit to make the matrix logically consistent, and then adjust other entries within acceptable deviations to make the matrix numerically consistent while preserving most of the original comparison information. In this paper, we firstly present some definitions and theories, based on which two effective methods are provided to identify directed circuits. Four optimization models are proposed to adjust the original inconsistent matrix. Finally, illustrative examples and comparison studies show the effectiveness and feasibility of our method.
\end{abstract}

Keywords: analytic hierarchy process (AHP), pairwise comparison matrix (PCM), logical inconsistency, numerical inconsistency, directed circuit.

DOI: $10.21629 /$ JSEE.2019.06.11

\section{Introduction}

Analytic hierarchy process (AHP) is a widely used methodology in many real-life decision-making problems $[1,2]$. Developed by Saaty in 1970s, AHP uses pairwise comparisons to capture relative importance of attributes, which forms the basis of priority determination. However, before a pairwise comparison matrix (PCM) can be used, it needs to pass a consistency test. If it fails to pass the test, the priority vector derived from the matrix would not be reliable. Therefore, it is necessary to improve the inconsis-

\footnotetext{
Manuscript received August 20, 2018.

*Corresponding author.

This work was supported by the National Natural Science Foundation of China (61601501; 61502521).
}

tency of a PCM.

Several approaches have been developed to aid the improving process. Some studies focus on reducing the consistent ratio (CR) as fast as possible [3,4], while the maximum revision element of the matrix usually becomes so large (e.g., changing an element from 6 to 3/5) that the original judgement of the decision maker (DM) could have been changed. Some other researchers have noticed this and made constraints to limit the maximum revision. For example, $\mathrm{Xu}$ and Wei [5] proposed an algorithm to derive a positive reciprocal matrix with acceptable consistency (i.e., $\mathrm{CR}<0.1$ ), gave the convergence theorem for the algorithm, and defined two indices to reflect the departure between the modified matrix and the original matrix. And Cao et al. [6] developed a heuristic approach to arrive at a consistent matrix that can retain more information than Xu and Wei [5] by comparing the two indices. They firstly decomposed the original matrix as the Hadamard product of a consistent matrix and a reciprocal deviation matrix, then constructed the modified matrix via a convex combination of the reciprocal deviation matrix and a zero deviation matrix, and gave the conditions under which the CR value keeps decreasing. Ergu et al. [7] also discussed a simple method to improve the consistency ratio of the PCM by introducing an induced matrix based on the original PCM, which is said to have preserved more information of the original PCM than existing techniques, but actually, the preservation of information is on fewer modified elements rather than the smallest total perturbation indices in $\mathrm{Xu}$ and Wei [5] and Cao et al. [6]. Pereira and Costa [8] proposed a nonlinear programming model in order to reduce inconsistency of a single judgment matrix to zero or near zero (i.e., $\mathrm{CR}=0$ ), which may result in more adjustment than needed. $\mathrm{Xu}$ and Wang [9] proposed a practical iterative algorithm to improve the consistency of complete and incomplete fuzzy preference relations that aim at retaining the original judgments as much as possible. Ashkan [10] developed a sampling-optimization-adjustment approach to im- 
prove consistency of positive reciprocal judgment matrices by optimizing their transformation into near-consistent matrices.

However, most of these studies have ignored the logical error in the PCM, where all elements in the PCM are equally treated, that is, limiting the modification range of each element equally so that the total deviation indices proposed by $\mathrm{Xu}$ and Wei [5] are kept as small as possible while $\mathrm{CR}$ is reduced below 0.1 . As a result, the logical error may still exist although the PCM has improved with $\mathrm{CR}<0.1$. In our opinion, if the entries are logically consistent, we assume the information represented by these entries logically correct, so we should only allow slight modifications to such entries to preserve most of the original information, e.g., change the element slightly from 5 to 4.8 . However, if some entries have logical errors, we should not obey this rule any more, in other words, these entries should be modified significantly to eliminate logical inconsistency, e.g., change from 5 to $1 / 5$.

Kou et al. [11] classified the inconsistency identification models for a PCM into three categories, cardinal inconsistency identification models, ordinal inconsistency identification models and models for both cardinal and ordinal inconsistency identification. They focused on the problem to improve both cardinal and ordinal inconsistencies and gave a good review of such problems. And they have proposed a Hadamard product induced bias matrix model to identify and adjust the cardinally inconsistent element(s) in a PCM, and used the adapted Hadamard model to identify and eliminate the ordinal inconsistencies. However, their model may fail to attain the goals of improving both cardinal and ordinal inconsistencies in some cases when the PCM is with high order (discussed in Example 2 in Section 4).

Different from traditional PCM, Yuen $[12,13]$ proposed a pairwise opposite matrix (POM) as an ideal alternative with respect to the human linguistic cognition of the rating scale of the paired comparison. He believes that the cognitive comparison of two objects should not be represented by their ratio $\left(a_{i j}=\omega_{i} / \omega_{j}\right)$ as human appears not to calculate multiplication or division by subjective measurement, e.g., height or weight comparison. Instead, the cognitive comparison of two objects should be represented by the difference between them $\left(b_{i j}=v_{i}-v_{j}\right)$. And, an accordant index (AI), similar to $\mathrm{CR}$, is defined to identify numerical consistency. However, these studies did not consider logical consistency, and how to improve when the POM is not with an acceptable consistency.

This research intends to discover a method that derives an acceptable consistency PCM $(\mathrm{CR}<0.1)$, eliminates all logical errors, and makes the departure between the modi- fied PCM and the original PCM even smaller than existed studies. The advantages of our method are mainly in that it is effective for high order PCM and PCM with element equal to 1 , suitable for very inconsistent situations, easy to realize by computer programming (no need to handle manually), and capable of obtaining optimum solution while ensure the modification for a single element within an acceptable range and the overall modification minimized. In this paper, we first identify the logical inconsistency elements by a method called directed circuits analysis, which finds all 3-node directed circuits in the matrix and generates the logical error elements accordingly. The elements with logical error will have larger modification bounds and not be included in the calculation of total deviation between the modified PCM and the original PCM. The other elements which are logically consistent will be modified slightly to preserve most correct information of the original PCM, and be included in the calculation of total deviation. Based on these principles, optimization models are built to derive an acceptable PCM.

The remainder of this paper is organized as follows. Firstly, we start with notations, definitions and theorems of the preference matrix, reachable matrix and directed circuits (Section 2). In Section 3, we present the idea and process of the consistency improving method, including logical inconsistency identification based on directed circuit analysis, and consistency improving strategy based on optimization models. Numerical examples are provided in Section 4 to illustrate and compare the proposed model. The paper is summarized and concluded in Section 5.

\section{Notations and definitions}

Fan and Xiao [14] proposed the preference matrix for the comparison matrix with linguistic entries, which is used to identify directed circuit in the comparison matrix. Similarly, we will extend the application of the preference matrix to the comparison matrix with other kinds of entries, such as one to nine scale entries, fuzzy reciprocal entries, interval number entries, POM entries [12], etc.

Suppose the decision making problem is to choose the optimum alternative from a set of alternatives, where $x_{i}$ represents the $i$ th alternative. The PCM is obtained by comparing any two alternatives among the alternative sets, for convenience, let $x_{i} \succ x_{j}$ represent $x_{i}$ superior to $x_{j}$, $x_{i} \sim x_{j}$ represent $x_{i}$ equal to $x_{j}$, and $x_{i} \prec x_{j}$ represent $x_{i}$ inferior to $x_{j}$.

Definition 1 A preference matrix $\boldsymbol{R}=\left[r_{i j}\right]_{n \times n}$ of the PCM $\boldsymbol{A}=\left[a_{i j}\right]_{n \times n}$ is defined as

$$
r_{i j}= \begin{cases}1, & x_{i} \succ x_{j} \\ 0, & \text { otherwise }\end{cases}
$$

where $\boldsymbol{R}$ can be generated by $\boldsymbol{A}$. For example, for one to 
nine scale entries, $r_{i j}=1$ holds when $a_{i j}>1$ is satisfied, otherwise $r_{i j}=0$; for fuzzy reciprocal entries, $r_{i j}=1$ holds when $a_{i j}>0.5$ is satisfied, otherwise $r_{i j}=0$; for linguistic entries, interval number entries and POM entries, $r_{i j}=1$ holds when $x_{i}$ is better than $x_{j}$, otherwise $r_{i j}=0$.

In this paper, we will mainly discuss the PCM with one to nine scale entries. Hence, when $x_{i}$ is better than $x_{j}$, we have $x_{i} \stackrel{a_{i j}}{\succ} x_{j}$, where the symbol " $\succ$ " denotes $x_{i}$ is $a_{i j}$ $\left(a_{i j}>1\right)$ times better than $x_{j}$; when $x_{j}$ is better than $x_{i}$, we have $x_{i} \stackrel{1 / a_{i j}}{\prec} x_{j}$, where the symbol " $\stackrel{1 / a_{i j}}{\prec}$ " denotes $x_{j}$ is $1 / a_{i j}\left(1 / a_{i j}>1\right)$ times better than $x_{i}$. In particular, when $x_{i}$ is equal to $x_{j}$, we have $x_{i} \sim x_{j}$. According to (1), $r_{i j}=1$ holds only when $x_{i} \succ^{a_{i j}} x_{j}$.

Definition 2 Given a preference matrix $\boldsymbol{R}=\left[r_{i j}\right]_{n \times n}$, a digraph based on $\boldsymbol{R}$ is defined as follows:

Use a circle with number $i$ inside to denote node $i$, where node $i$ represents $x_{i}(i=1,2, \ldots, n)$. As shown in Fig. 1, there are three nodes 1, 2, 3 representing $x_{1}, x_{2}$, $x_{3}$ respectively. If $x_{i} \succ x_{j}$, i.e., $r_{i j}=1$ by (1), we draw a directed arc from node $i$ to node $j$, and if $x_{i} \sim x_{j}$, a dashed line is drawn between node $i$ and node $j$ (see Fig. 2(a)). If each $r_{i j}$ is represented as a directed arc, a digraph based on $\boldsymbol{R}$ is constructed. For example, a $3 \times 3$ PCM $\boldsymbol{A}$ and its corresponding preference matrix $\boldsymbol{R}$, and the digraph based on $\boldsymbol{R}$ are shown in Fig. 1.

$$
\boldsymbol{A}=\left[\begin{array}{ccc}
1 & 3 & 5 \\
1 / 3 & 1 & 1 / 2 \\
1 / 5 & 2 & 1
\end{array}\right] \quad \boldsymbol{R}=\left[\begin{array}{lll}
0 & 1 & 1 \\
0 & 0 & 0 \\
0 & 1 & 0
\end{array}\right]
$$

Fig. 1 An example to illustrate $R$ and the digraph based on $R$

$$
\boldsymbol{A}=\left[\begin{array}{ccc}
1 & 3 & 5 \\
1 / 3 & 1 & 1 \\
1 / 5 & 1 & 1
\end{array}\right] \quad \boldsymbol{R}=\left[\begin{array}{lll}
0 & 1 & 1 \\
0 & 0 & 0 \\
0 & 0 & 0
\end{array}\right] \quad(1)
$$

(a) PCM with elements equal to 1 except the main diagonal

$$
\boldsymbol{A}=\left[\begin{array}{ccc}
1 & 3 & 5 \\
1 / 3 & 1 & 0.999 \\
1 / 5 & 1.000 & 1
\end{array}\right] \quad \boldsymbol{R}=\left[\begin{array}{lll}
0 & 1 & 1 \\
0 & 0 & 0 \\
0 & 1 & 0
\end{array}\right]
$$

(b) Equivalent PCM to $\boldsymbol{A}$ by Assumption 1

Fig. 2 An example of PCM with elements equal to 1

The digraph based on the preference matrix is a very special digraph with the following two features, which forms the basis of the 3-node directed circuit method discussed in Section 3.1.

(i) The minimum directed circuit has three nodes, which is called 3-node directed circuit, or three-way cycle [15]. There are no single node directed circuits (self-loopback node), and no 2-node directed circuits because there are no bidirectional arrows in the digraph.

The number of minimum directed circuits $c$ can be derived from the preference matrix $\boldsymbol{R}=\left[r_{i j}\right]_{n \times n}$ by Du [16] as follows:

$$
c=\frac{1}{6} n(n-1)(n-2)-\frac{1}{2} \sum_{i=1}^{n} h_{i}\left(h_{i}-1\right)
$$

where $h_{i}=\sum_{j=1}^{n} r_{i j}$.

(ii) Pairwise unidirectional connectivity. In accordance with the connectivity of the preference matrix $\boldsymbol{R}$, for any two nodes $i$ and $j$, either $x_{i} \succ x_{j}$ or $x_{i} \precsim x_{j}$ is satisfied, which means there should exist a unidirectional arrow or a dashed line between nodes $i$ and $j$. Hence, we can conclude that the digraph based on the preference matrix is very special in that it only has unidirectional arrows, and no bidirectional arrows. It is obvious that the total number of unidirectional arrows for a digraph based on preference matrix $\boldsymbol{R}=\left[r_{i j}\right]_{n \times n}$ is $n(n-1) / 2-n_{e q}$, where $n_{e q}$ represents the total number of elements equal to 1 in the upper diagonal triangular matrix of the PCM (except the main diagonal). For example, there are three unidirectional arrows in Fig. 1, and two in Fig. 2(a).

However, elements equal to 1 will destroy the pairwise unidirectional connectivity, e.g., we cannot draw a unidirectional arrow between node 2 and node 3 in Fig. 2(a). To make the problem easier to handle, similar to Kou et al. [11], we give the following assumption.

Assumption 1 For a given PCM $\boldsymbol{A}=\left[a_{i j}\right]_{n \times n}$, the equivalent no one element PCM (NOE-PCM) $\boldsymbol{A}^{\prime}=$ $\left[a_{i j}^{\prime}\right]_{n \times n}$ is defined as

$$
a_{i j}^{\prime}=\left\{\begin{array}{l}
0.9999 \text { or } 1.0001, \quad a_{i j}=1 ; i<j \\
a_{i j}, \quad a_{i j} \neq 1 ; i<j \\
\frac{1}{a_{j i}^{\prime},} \quad i>j \\
1, \quad i=j
\end{array} .\right.
$$

Obviously, $\boldsymbol{A}^{\prime}$ is a PCM since 1.0001 and 0.9999 are in the feasible region of $[1 / 9,9]$, and they are reciprocal. With the slight modification by Assumption 1, we can draw a unidirectional arrow between any two nodes in $\boldsymbol{A}^{\prime}$ (e.g., the diagraph in Fig. 2(b)), and $\boldsymbol{A}^{\prime}$ will not change the final decision numerically, e.g., $\boldsymbol{A}^{\prime}$ and $\boldsymbol{A}$ in Fig. 2 have the same priority vectors $\boldsymbol{\omega}=\{0.6586,0.1852,0.1562\}$, and the same priority order $1 \succ 2 \succ 3$. Note that the elements that equal 1 can be set to 0.9999 or 1.0001 depending on whether the modified NOE-PCM has the least 3-node directed circuits, in other words, has minimum $c$ in (2). Thus we need to decide which modification is the best choice. 
Take the PCM $\boldsymbol{A}$ in Fig. 2(a) for example, since there is only one element equal to 1 , we have two choices of the modified NOE-PCM, i.e., change $a_{23}$ to 0.9999 or 1.0001 . By comparing these two PCMs, $a_{23}=0.9999$ should be the better choice with no 3-node directed circuits inside $(c=0)$. Therefore, NOE-PCM is almost equal to the original PCM in numerical consistency, while the logical consistency can be reduced. This is a simple example easy to handle, but in more cases, there are more than one element equal to 1 , e.g., $n_{1}$ elements, then we will have $2^{n_{1}}$ different combinations of possible NOE-PCMs. For example, in Example 4 in Section 4, $n_{1}=9$, there should be $2^{9}=$ 512 different cases of the modified NOE-PCMs, and we only choose the modified NOE-PCM with minimum logical inconsistency, i.e., minimum $c$ in (2), see Example 4 for details.

In the remainder of this paper, we assume that the PCM is an NOE-PCM. For a PCM with elements equal to 1, we will firstly transform it to an equivalent NOE-PCM using (3).

Specifically, it is logical that three equivalent elements equal 1 and form a circle in a matrix. Obviously, we can infer that these three priorities are equal. Our method can also handle this situation easily. See the example below, for the PCM $\boldsymbol{A}$ in Fig. 3, there should be $2^{3}=8$ different cases of equivalent NOE-PCMs, and we choose the modified NOE-PCM $\boldsymbol{A}^{\prime}$ with $c=0$. We also find that for $\boldsymbol{A}^{\prime}$, $\mathrm{CR}=0$ and $\boldsymbol{\omega}=\{1 / 3,1 / 3,1 / 3\}$, which means it is both logical and numerically consistent, and these three priorities are equal.

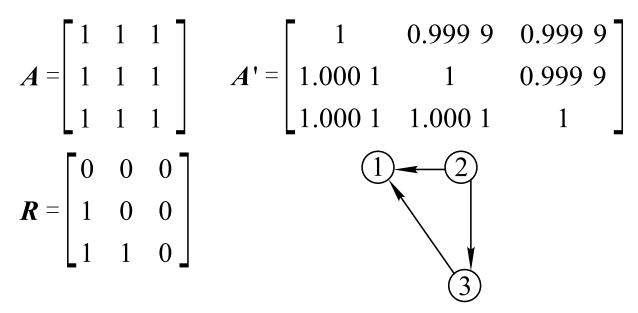

Fig. 3 Example of PCM with three elements equal to 1

Definition 3 [14] Given a preference matrix $\boldsymbol{R}=$ $\left[r_{i j}\right]_{n \times n}$, the reachable matrix $\boldsymbol{T}$ is defined as

$$
\boldsymbol{T}=\boldsymbol{R} \dot{+} \boldsymbol{R}^{2} \dot{+} \cdots \dot{+} \boldsymbol{R}^{n}
$$

where the power of $\boldsymbol{R}$ follows matrix multiplication rules, and $\dot{+}$ is the Boolean sum, i.e., $0 \dot{+} 0=0,0 \dot{+} 1=1$, $1 \dot{+} 0=1,1 \dot{+} 1=1$.

Definition 4 The nodes set $\left\{u_{1}, u_{2}, \ldots, u_{m}\right\}$ is said to form a directed circuit, if there exist $m$ elements in the preference matrix $\boldsymbol{R}=\left[r_{i j}\right]_{n \times n}$ that satisfy $m \geqslant 3$ and $x_{u 1} \succ x_{u 2} \succ \cdots \succ x_{u m} \succ x_{u 1}$.
Definition 5 For a given PCM $\boldsymbol{A}=\left[a_{i j}\right]_{n \times n}, \boldsymbol{A}$ is called logically consistent, if there is no directed circuit in its preference matrix $\boldsymbol{R}$. Otherwise, if there exist any directed circuits, $\boldsymbol{A}$ is called a logically inconsistent matrix, and the elements that form directed circuits are called logically inconsistent elements, while the other elements not belong to any directed circuit are called logically consistent elements.

Note that logical consistency is quite similar to ordinal consistency (or transitivity) in [17] and weak consistency in [18]. If $x_{i} \succ x_{j} \succ x_{k}$, then $x_{i} \succ x_{k}$ should be satisfied. If however, $x_{k} \succ x_{i}$ when $x_{i} \succ x_{j} \succ x_{k}$, then the preference judgments are called logically inconsistent, or intransitivity. Therefore, the logical inconsistency can be defined as $x_{i} \succ x_{j} \succ x_{k} \succ x_{i}$, which represents a directed circuit. Actually, logical consistency requires the DM to be consistent for each pairwise comparison, which may be unrealistic in real-life applications, especially for high order PCM.

Definition 6 For a given PCM $\boldsymbol{A}=\left[a_{i j}\right]_{n \times n}$, if $\mathrm{CR}<0.1, \boldsymbol{A}$ is said to have acceptable numerical consistency.

Definition 7 For a given PCM $\boldsymbol{A}=\left[a_{i j}\right]_{n \times n}$, we call $\boldsymbol{A}$ has reached acceptable consistent level, if and only if $\boldsymbol{A}$ has acceptable numerical consistency and its preference matrix $R$ is logically consistent.

Theorem 1 The PCM is logically consistent, if there exist no directed circuits in its corresponding preference matrix.

This theorem can be easily derived from Definition 5 .

Corollary 1 The PCM is logically consistent, if $c=0$ holds, where $c$ is obtained by preference matrix using (2).

Proof If $c=0$ holds, which means the number of minimum directed circuits is 0 , in other words, there exist no directed circuits in its corresponding preference matrix. According to Theorem 1, the PCM is logically consistent.

Some researchers [14] consider logical consistency as the acceptable consistent level, while some others $[5,6]$ consider numerical consistency as the acceptable consistent level. In our opinion, both ideas are one-sided. In fact, for a PCM, logical consistency cannot guarantee acceptable numerical consistency, e.g., $\boldsymbol{A}_{1}$ is a PCM, and we cannot find any directed circuit in its corresponding preference matrix, which means $\boldsymbol{A}_{1}$ is logically consistent according to Definition 5; while $\mathrm{CR}\left(\boldsymbol{A}_{1}\right)=0.1617>0.1$, which means $\boldsymbol{A}_{1}$ is acceptable in numerical consistency according to Definition 6. On the contrary, acceptable numerical consistency cannot guarantee logically consistency either, e.g., $\boldsymbol{A}_{2}$ is as follows, and $\mathrm{CR}\left(\boldsymbol{A}_{2}\right)=0.0895<0.1$, which means $\boldsymbol{A}_{2}$ has acceptable numerical consistency, but 
it is logically inconsistent since $x_{5} \succ x_{3} \succ x_{1} \succ x_{5}$. In our opinion, both $\boldsymbol{A}_{1}$ and $\boldsymbol{A}_{2}$ are unacceptable. Generally speaking, logical consistency and acceptable numerical consistency do not have necessary relationships. However, in most cases, a logically inconsistent PCM is usually unacceptable in numerical consistency.

$$
\begin{gathered}
\boldsymbol{A}_{1}=\left[\begin{array}{cccc}
1 & 2 & 5 / 2 & 5 \\
1 / 2 & 1 & 9 & 2 \\
2 / 5 & 1 / 9 & 1 & 1 / 2 \\
1 / 5 & 1 / 2 & 2 & 1
\end{array}\right] \\
\boldsymbol{A}_{2}=\left[\begin{array}{ccccc}
1 & 9 / 2 & 2 / 3 & 2 & 3 / 2 \\
2 / 9 & 1 & 1 / 5 & 3 / 2 & 1 / 2 \\
3 / 2 & 5 & 1 & 11 / 2 & 1 / 2 \\
1 / 2 & 2 / 3 & 2 / 11 & 1 & 1 / 5 \\
2 / 3 & 2 & 2 & 5 & 1
\end{array}\right]
\end{gathered}
$$

Theorem 2 Each directed circuit in the digraph created by the preference matrix can be decomposed to 3-node directed circuits.

Proof For a given preference matrix $\boldsymbol{R}=\left[r_{i j}\right]_{n \times n}$, suppose that there exists a $k$-node directed circuit $x_{1} \succ$ $x_{2} \succ x_{3} \succ \cdots \succ x_{k-1} \succ x_{k} \succ x_{1}$, as shown in Fig. 4(a). Assume that $x_{1} \succ x_{k-1}$, thus a directed arc from node 1 to node $k-1$ is drawn, and a 3 -node directed circuit $x_{1} \succ x_{k-1} \succ x_{k} \succ x_{1}$ is generated, see Fig. 4(b). Otherwise, assume that $x_{1} \precsim x_{k-1}$, then we can draw a directed arc from node $k-1$ to node 1 , and we obtain a $(k-1)$-node directed circuit $x_{1} \succ x_{2} \succ x_{3} \succ \cdots \succ x_{k-1} \succ x_{1}$, see Fig. 4(c). For this $(k-1)$-node directed circuit, continue to assume relationship between $x_{2}$ and $x_{k-1}$, and we will obtain either a $(k-2)$-node directed circuit, or a 3-node directed circuit. And repeat this process, suppose that there is no 3-node directed circuit generated during the process, then we will obtain $(k-1)$-node, $(k-2)$-node, $\ldots, 4$-node directed circuits successively, and for the 4-node directed circuit, Fig. 5 shows that we can always generate a 3 -node directed circuit.

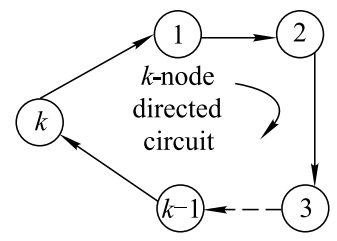

(a) $k$-node directed circuit

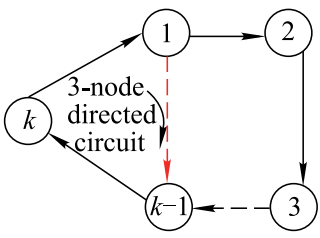

(b) $x_{1} \succ x_{k-1}, 3$-node circuit generated

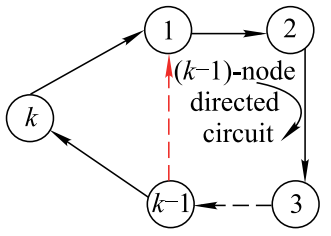

(c) $x_{1} \prec x_{k-1},(k-1)$-node circuit generated

Fig. $4 k$-node directed circuit decomposing
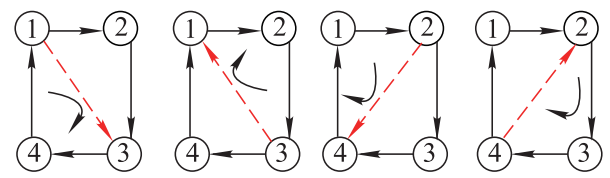

Fig. 5 4-node directed circuit decomposing

Corollary 2 If there exist any directed circuits in a digraph based on the preference matrix, it is certain that we can find at least one 3-node directed circuit in the digraph, and the 3-node directed circuits are the main factors that cause logical inconsistency. In other words, the logical inconsistency is mainly caused by the 3-node directed circuits in the preference matrix.

Hence, by Corollary 2, we can firstly use some existing methods to find all possible directed circuits in a digraph, and then only pay attention to the 3-node directed circuits among them.

Lemma 1 [16] $\quad \boldsymbol{R}^{k}=\left[r_{i j}^{k}\right]_{n \times n}(k>1)$ represents the $k$ th power of matrix $\boldsymbol{R}$, which is calculated following Boolean operation rules. If the element $r_{i j}^{k}=1$, it means there exist at most $k$ and at least 1 directed arcs from node $i$ to node $j$; otherwise, if $r_{i j}^{k}=0$, it means there exist no directed arcs from node $i$ to node $j$.

Theorem 3 The preference matrix $\boldsymbol{R}=\left[r_{i j}\right]_{n \times n}$ is logically inconsistent, if there exist element(s) equal to 1 on the main diagonal of its reachable matrix $\boldsymbol{T}=\left[t_{i j}\right]_{n \times n}$, where $\boldsymbol{T}$ is obtained by $\boldsymbol{R}$ using (4). Otherwise, if each element on the main diagonal of $\boldsymbol{T}$ equals $0, \boldsymbol{R}$ is logically consistent.

Proof By Definition 3, we have $t_{i i}=r_{i i} \dot{+} r_{i i}^{2} \dot{+} \cdots$ $\dot{+} r_{i i}^{n}$, where $r_{i i}^{k}$ is the element of matrix $\boldsymbol{R}^{k}$. Suppose $t_{i i}=1(i \in\{1,2, \ldots, n\})$, it is certain that there exists a $r_{i i}^{k}=1(1<k \leqslant n)$ since $r_{i i}=0$ by (1). By Lemma 1, there is at least one directed circuit from node $i$ to node $i$ because the directed arc shares the same start point and end point: node $i$. It can be observed from Definition 5 that $\boldsymbol{R}$ is logically inconsistent. On the contrary, if $t_{i i}=0(\forall i, 1 \leqslant i \leqslant n)$, we have $r_{i i}^{k}=0$ $(\forall k, i, 1<k \leqslant n, 1 \leqslant i \leqslant n)$. By Lemma 1 , for each node $i$, there exist no directed arcs from node $i$ to node $i$, in other words, there exist no directed circuits, which means $\boldsymbol{R}$ is logically consistent by Definition 5 .

By Theorem 3, we can easily tell whether a preference matrix $\boldsymbol{R}$ is logically consistent or not. However, we do 
not know which elements have caused the logical inconsistency, and which nodes have formed the directed circuits. Hence, we propose Corollary 3 and Theorem 4 to help us identify the elements that may have logical errors.

Corollary 3 If there exists any elements equal to 1 on the main diagonal of reachable matrix $\boldsymbol{T}=\left[t_{i j}\right]_{n \times n}$, there should exist at least three or more elements equal to 1 on the main diagonal of $\boldsymbol{T}$.

Proof By Theorem 2, if there exist directed circuits in a digraph based on the preference matrix, we can definitely decompose the circuits to 3-node directed circuits. By Theorem 3 , the element $t_{i i}=1(i=1,2, \ldots, n)$ means node $i$ is on a directed circuit, in other words, there must exist at least one directed circuit. According to the feature of digraph based on the preference matrix, the minimum directed circuit has three nodes, which means there should be at least three nodes on a directed circuit, as well as three elements equal to 1 on the main diagonal of $\boldsymbol{T}$ besides node $i$.

Theorem 4 If there exists any element equal to 1 on the main diagonal of reachable matrix $\boldsymbol{T}=\left[t_{i j}\right]_{n \times n}$, it is certain that there exist directed circuits generated by the nodes corresponding to these elements.

Proof Suppose $t_{i i}=1(i \in\{1,2, \ldots, n\})$, by Theorem 3 , there is at least one directed circuit from node $i$ to node $i$. And by Theorem 2, the directed circuit in the digraph created by the preference matrix can be decomposed to 3-node directed circuits, to be clear, we suppose the nodes $\{i, j, l\}$ form the directed circuit $i \rightarrow j \rightarrow l \rightarrow i$. If we start the circle from node $j$, then the directed circuit is $j \rightarrow l \rightarrow i \rightarrow j$, which means $t_{j j}=1$ according to Theorem 3 and Lemma 1. Similarly, we can prove $t_{l l}=1$. That is, the directed circuits are generated by those nodes that satisfy $t_{i i}=1(i \in\{1,2, \ldots, n\})$.

Theorem 5 If each element on the main diagonal of reachable matrix $\boldsymbol{T}$ equals 0 , the priority order can be determined by comparing the number of ones on each row or column of the preference matrix $\boldsymbol{R}$, and the more elements equal to 1 , the better priority order.

Proof If each element on the main diagonal of $\boldsymbol{T}$ equals 0 , by Theorem 3 , the PCM is logically consistent, and no directed circuits exist in $\boldsymbol{R}$, by Theorem 1 . Therefore, we can conclude that $x_{i}$ is better than $x_{j}$ if $r_{i j}>1$, since no logical inconsistency exists. Suppose row $i$ is with $k$ ones, that is, there should be $k$ elements in $\boldsymbol{R}$ that satisfy $r_{i j}>1(j \in\{1,2, \ldots, n\})$, which means $x_{i}$ is better than the other $k-1$ alternatives. By this rule, we can easily conclude that the more ones in row $i$, the better priority order row $i$ should be.

By Theorem 5, we can determine the priority order for a logically consistent PCM as follows.
Firstly, summing up each row, we have

$$
S_{i}=\sum_{j=1}^{n} r_{i j}
$$

where $S_{i}$ is the sum of row $i$ of the preference matrix.

Then, sort $S_{i}$ in descending order to obtain the priority order from superior to inferior.

If we only want to determine the priority order instead of the priority vector, Theorem 5 and (5) are qualified and easy to use, as well as very applicable to different kinds of entries, such as one to nine scale entries, fuzzy reciprocal entries, interval number entries, POM entries [12,13], etc., if only the preference matrix can be obtained by (1).

However, there is an apparent disadvantage for this method when two rows or columns have the same $S_{i}$. In such cases, we cannot determine the priority order by just observing the preference matrix. Instead, we must use the information of the PCM.

Theorem 6 [19] Let $\boldsymbol{A}=\left[a_{i j}\right]_{n \times n}$ be an inconsistent PCM with $\mathrm{CR}(\boldsymbol{A})>0.1$, then there must exist one element $a_{i j}$ that can make $\operatorname{CR}(\boldsymbol{A})$ decrease when $a_{i j}$ is modified.

By Theorem 6, we can conclude that the $C R$ value can keep decreasing by modifying proper elements of the PCM, which forms the theoretical basis of our optimization models in Section 3.2.1.

\section{Consistency improving method}

\subsection{Logical consistency identification based on directed circuit analysis}

The priority order of the alternatives will be questionable when the PCM is logically inconsistent. In fact, if the PCM is logically inconsistent, different prioritization methods, such as cosine maximization method (CM) [20], eigenvector method (EV) [21], weighted least squares method (WLS) [22], additive normalization method (AN) [23], logarithmic least squares method (LLS) [24], etc., will obtain different prioritization orders (see Example 2 in [20]). As a result, logical inconsistency should be identified and eliminated firstly so that the priority order and the weighting results can be determined. Two methods are proposed here to help the DMs to identify logical inconsistencies.

(i) Reachable matrix method

By Theorem 3 and Theorem 4, we give the identification method by analyzing the reachable matrix.

Step 1 Calculate the reachable matrix $T$ using (4);

Step 2 Find all nodes that satisfy $t_{i i}=1(i \in$ $\{1,2, \ldots, n\})$, and suppose there are $m$ such nodes $\left\{i_{1}, i_{2}, \ldots, i_{m}\right\}$ where $m \geqslant 3$ (by Corollary 3 );

Step 3 Select three nodes from $\left\{i_{1}, i_{2}, \ldots, i_{m}\right\}$ and analyze whether these three nodes form a directed cir- 
cuit. Suppose that we have selected $\left\{i_{p}, i_{q}, i_{l}\right\}$, then these three elements $a_{p q}, a_{q l}, a_{p l}$ in $\boldsymbol{A}$ will need to make comparisons. For example, if $x_{p}{\stackrel{a_{p q}}{\succ}}^{x_{q}} \stackrel{a}{q l}^{\succ} x_{l} \stackrel{1 / a_{p l}}{\succ} x_{p}$ or $x_{p} \stackrel{1 / a_{p q}}{\prec} x_{q} \stackrel{1 / a_{q l}}{\prec} x_{l} \stackrel{a_{p l}}{\prec} x_{p}$ holds, the three nodes $\left\{i_{p}, i_{q}, i_{l}\right\}$ make a 3-node directed circuit. We will need to make such analysis for $\mathrm{C}_{m}^{3}$ times to cover all possible 3-node directed circuit.

This method is simple and effective when $m$ is small. For example, when $m=3$, only one judgment $\left(\mathrm{C}_{3}^{3}=1\right)$ is required (see Example 1 and Example 4 in Section 4), with the three elements equal to 1 on the main diagonal of the reachable matrix making a directed circuit; when $m=4$, we need four judgments $\left(\mathrm{C}_{4}^{3}=4\right)$ manually (see Example 2 in Section 4). Take Example 1 in [17] as an illustration (see Fig. 6). We find that the PCM has a consistency ratio of 0.083 , which means the PCM is numerically acceptable, but the reachable matrix $T$ has five elements equal to 1 on the main diagonal $(m=5)$, and the number of minimum directed circuits $c=4$ according to the preference matrix $\boldsymbol{R}$ by (2), which means there should exist four 3 -node directed circuits in the 10 potential judgments $\left(\mathrm{C}_{5}^{3}=10\right)$, and the PCM is logically unacceptable. However, as $m$ becomes greater, e.g., $m=7$, we need 35 judgments $\left(\mathrm{C}_{7}^{3}=35\right)$ manually, which makes the method too time consuming. Therefore, when $m \geqslant 5$, we recommend to use the 3-node directed circuit method, which is easy to realize by computer programming and can find the results fast and accurately. The 3-node directed circuit method is discussed as follows.

$$
\begin{gathered}
\boldsymbol{A}=\left[\begin{array}{ccccc}
1 & 7 / 4 & 3 / 4 & 5 / 2 & 7 / 4 \\
4 / 7 & 1 & 3 / 4 & 9 / 4 & 9 / 4 \\
4 / 3 & 4 / 3 & 1 & 3 / 4 & 3 / 4 \\
2 / 5 & 4 / 9 & 4 / 3 & 1 & 5 / 8 \\
4 / 7 & 4 / 9 & 4 / 3 & 8 / 5 & 1
\end{array}\right] \\
\boldsymbol{R}=\left[\begin{array}{lllll}
0 & 1 & 0 & 1 & 1 \\
0 & 0 & 0 & 1 & 1 \\
1 & 1 & 0 & 0 & 0 \\
0 & 0 & 1 & 0 & 0 \\
0 & 0 & 1 & 1 & 0
\end{array}\right] \quad \boldsymbol{T}=\left[\begin{array}{ccccc}
1 & 1 & 1 & 1 & 1 \\
1 & 1 & 1 & 1 & 1 \\
1 & 1 & 1 & 1 & 1 \\
1 & 1 & 1 & 1 & 1 \\
1 & 1 & 1 & 1 & 1
\end{array}\right]
\end{gathered}
$$

Fig. 6 Preference matrix $R$ and reachable matrix $T$ for Example 1 in [17]

(ii) 3-node directed circuit method

This method firstly generates all possible directed circuits in the digraph by the method in [25], among which all the 3-node directed circuits are selected according to Theorem 2.

Step 1 Convert the preference matrix $\boldsymbol{R}=\left[r_{i j}\right]_{n \times n}$ to edge matrix $\boldsymbol{E}=\left[e_{i j}\right]_{n \times n}$ of the digraph. Let $\boldsymbol{E}(k)=\boldsymbol{E}$, $k=1$.

$$
e_{i j}=\left\{\begin{array}{l}
v_{i} v_{j}, \quad r_{i j}=1 \\
\phi, \quad r_{i j}=0
\end{array}\right.
$$

where $v_{i} v_{j}$ denotes the edge or directed arc from node $v_{i}$ to $v_{j}$. For $k=1$, we have

$$
\boldsymbol{E}(1)=\left[\begin{array}{cccc}
e_{11} & e_{12} & \cdots & e_{1 n} \\
e_{21} & e_{22} & \cdots & e_{2 n} \\
\vdots & \vdots & \ddots & \vdots \\
e_{n 1} & e_{n 2} & \cdots & e_{n n}
\end{array}\right] .
$$

Step 2 Calculate the path matrix $\boldsymbol{W}(k)$ by

$$
\boldsymbol{W}(k)=\boldsymbol{E}_{c}(k) \cdot \boldsymbol{E}_{r}(k)
$$

where $\boldsymbol{E}_{c}(k)$ is a column vector from the second element to the last element in the first column of $\boldsymbol{E}(k)$, and $\boldsymbol{E}_{r}(k)$ is a row vector from the second element to the last element in the first row of $\boldsymbol{E}(k)$. For example, $\boldsymbol{E}_{c}(1)=$ $\left[e_{21}, e_{31}, \ldots, e_{n 1}\right]^{\mathrm{T}}, \boldsymbol{E}_{r}(1)=\left[e_{12}, e_{13}, \ldots, e_{1 n}\right]$, and we have

$$
\boldsymbol{W}(1)=\left[\begin{array}{cccc}
e_{21} e_{12} & e_{21} e_{13} & \cdots & e_{21} e_{1 n} \\
e_{31} e_{12} & e_{31} e_{13} & \cdots & e_{31} e_{1 n} \\
\vdots & \vdots & \ddots & \vdots \\
e_{n 1} e_{12} & e_{n 1} e_{13} & \cdots & e_{n 1} e_{1 n}
\end{array}\right]
$$

where the multiplication of edges is defined as follows. Suppose $e_{i j}=v_{i 1} v_{i 2} \ldots v_{i r}$, and $e_{q l}=v_{j 1} v_{j 2} \ldots v_{j k}$, we define

$$
e_{i j} e_{q l}=\left\{\begin{array}{c}
v_{i 1} v_{i 2} \cdots v_{i r} v_{j 2} v_{j 3} \cdots v_{j k} \\
v_{i r}=v_{j 1} ; e_{i j} \cap e_{q l} \in\left\{\left\{v_{i r}\right\}\right. \\
\left.\left\{v_{i r}, v_{i 1}=v_{j k}\right\}\right\} \\
\phi, \quad \text { otherwise }
\end{array} .\right.
$$

Step 3 If there exist non-empty elements on the main diagonal of $\boldsymbol{W}(k)$, the paths represented by such elements will create directed circuits. We record such elements in the recorded circuits set.

Step 4 Modify the edge matrix $\boldsymbol{E}(k)$. Let $k=k+1$, if $k=n-1$ is satisfied, go to Step 5; otherwise, let

$$
\boldsymbol{E}(k)=\boldsymbol{W}(k-1)+\boldsymbol{E}^{\prime}(k-1)
$$

where $\boldsymbol{E}^{\prime}(k-1)$ is a matrix derived from $\boldsymbol{E}(k-1)$ with the first row and first column deleted, and go to Step 2. For example, if $k=2$, we have

$$
\boldsymbol{E}^{\prime}(1)=\left[\begin{array}{cccc}
e_{22} & e_{23} & \cdots & e_{2 n} \\
e_{32} & e_{33} & \cdots & e_{3 n} \\
\vdots & \vdots & \ddots & \vdots \\
e_{n 2} & e_{n 3} & \cdots & e_{n n}
\end{array}\right]
$$

Note that the sum operation of edge matrices is defined as follows. Let $e_{i j}=v_{i 1} v_{i 2} \cdots v_{i r}, e_{q l}=v_{j 1} v_{j 2} \cdots v_{j k}$, we have

$$
e_{i j}+e_{q l}=\left\{v_{i 1} v_{i 2} \cdots v_{i r}, v_{j 1} v_{j 2} v_{j 3} \cdots v_{j k}\right\} .
$$


Step 5 Select all the 3-node directed circuits from the recorded circuits set, and end the procedure.

We have noted that Kou et al. [11] also proposed methods for identifying the directed circuits based on the theorem of the Hadamard product induced bias matrix (HPIBM). Kou's method can identify the directed circuits effectively with some manual analysis involved, which is similar to the reachable matrix method, thus it may be less effective to deal with a PCM with higher order and many logical inconsistencies. Compared with Kou's and reachable matrix methods, the 3 -node directed circuit method is easier to realize through computer programming, since no manual analysis is required.

\subsection{Consistency improving method based on optimization}

The foregoing arguments show that the 3-node directed circuits in the preference matrix are the main causes of logical inconsistency. The elements of the PCM related to the 3node directed circuits are required to be modified to attain the acceptable consistent level as defined in Definition 7. In this section, we will solve this problem by the optimization method.

\subsubsection{Optimization model}

By Definition 7, we assume that the acceptable consistent level means that the PCM should be both logically and numerically consistent. In our earlier studies [26], we have proposed an optimization model to attain numerical consistency while preserve most of the original comparison information. Similar studies, such as [5] and [6], also have discussed such issue. Since logical consistency is ignored, and the modification range of each element in the original PCM is limited in order to preserve original comparison information, it is possible that the modified PCM remains logical inconsistency because greater modifications are required for reaching logical consistency, e.g., change from 5 to $1 / 5$ instead of a number in the modification range [4.5, 6]. Therefore, we have to treat the elements' modification range differently: for those logically inconsistent elements, we allow a much greater modification range; for those logically consistent elements, we control the range of modification to realize minimum changes so that most of these comparison information can be preserved.

(i) Model 1: Consider the minimization of $\mathrm{CR}$ as an objective function, and the acceptable consistent level as constraints. Let $\left\{x_{1}, x_{2}, \ldots, x_{m}\right\}$ denote all elements in the 3-node directed circuit, which only consider the elements in the upper diagonal triangular matrix of PCM. Let $y_{i}$ denote elements with logical error, note that only elements in the 3-node directed circuit are possibly logically incor- rect, $y_{i} \in\left\{x_{1}, x_{2}, \ldots, x_{m}\right\}$. However, not all elements in a 3-node directed circuit are with logical error, but possibly one or two. In general, for a 3-node directed circuit, we should redirect one or two directed arcs to eliminate the directed circuit. The redirection of $y_{i}$ is defined in the following.

$$
\begin{aligned}
& \min _{x_{1}, x_{2}, \ldots, x_{m}}\{\mathrm{CR}\} \\
& \text { s.t. } y_{i} \in\left\{\begin{array}{l}
{[1 / 9,0.9999], \quad y_{i}>1} \\
{[1.0001,9], \quad y_{i}<1}
\end{array}\right. \\
& \operatorname{CR}(\boldsymbol{C}) \leqslant 0.1
\end{aligned}
$$

where $y_{i}$ denotes elements with logical error $\left(y_{i} \neq 1\right.$ by Assumption 1), and we need to redirect $y_{i}$, that is, when $y_{i}>1$, we search for the modified $y_{i}$ within [1/9, 0.999 9]; when $y_{i}<1$, we search for the modified $y_{i}$ within [1.000 1,9], where 0.9999 and 1.0001 are used to avoid $y_{i}=1$ to prevent new logical error from being generated. $C$ denotes the modified PCM, and $\mathrm{CR}(\boldsymbol{C})$ is the consistent ratio of C.

(ii) Model 2: This optimization model has the same objective function and numerical consistent constraint as Model 1. Here, we allow all elements in the 3-node directed circuit to be modified within [1/9,9], while modify other elements $c_{i j}$ within a given bound.

$$
\begin{gathered}
\min _{x_{1}, x_{2}, \ldots, x_{m}, c_{i j}}\{\mathrm{CR}\} \\
\text { s.t. } c_{i j} \in\left[\max \left\{1.0001, a_{i j}-\delta\right\}, \min \left\{a_{i j}+\delta, 9\right\}\right], \\
c_{i j} \notin\left\{x_{1}, x_{2}, \ldots, x_{m}\right\} ; c_{i j} \geqslant 1 \\
x_{i} \in[1 / 9,9], \quad \forall i \in\{1,2, \ldots, m\} \\
\operatorname{CR}(\boldsymbol{C}) \leqslant 0.1
\end{gathered}
$$

where $x_{i}$ denotes element in the 3-node directed circuit, we search for the modified $x_{i}$ within $[1 / 9,9]$, i.e., the whole feasible range, which means we ignore the original information of the elements with possible logical error and make the matrix incomplete. $c_{i j}$ denotes the elements not belong to any 3 -node directed circuit. $\delta$ is the allowable deviation of $c_{i j}$, and $\delta$ must be less than 1 so that the modified element is within the tolerance deviations intervals [19], which are defined in Table 1.

Table 1 Linguistic description of scale and its corresponding tolerance deviations interval

\begin{tabular}{ccc}
\hline Linguistic description & Scale & $\begin{array}{c}\text { Tolerance } \\
\text { deviations } \\
\text { interval }\end{array}$ \\
\hline Extreme importance & 9 & {$[8,10]$} \\
Demonstrated importance & 7 & {$[6,8)$} \\
Strong importance & 5 & {$[4,6)$} \\
Moderate importance & 3 & {$[2,4)$} \\
Equal importance & 1 & {$[1,2)$} \\
\hline
\end{tabular}


For example, when $i$ is strongly important to $j$, the scale of $a_{i j}$ is 5 , and the tolerance deviations interval is [4,6), i.e., $\delta=1$, which means $a_{i j}$ can be substituted by any real number on the interval $[4,6)$. By using allowable deviation $\delta$, we actually extend the integer number ranging from 1 to 9 to an acceptable interval. Also, Fig. 7 gives the corresponding relationship between tolerance deviations intervals and linguistic descriptions of importance scales. Obviously, the smaller $\delta$ is, the more information the modified PCM can preserve. In this paper, we set $\delta_{\max }$ to be 1 . To avoid out of bound, we set $c_{i j}$ to be the near bound when it is out of $[1 / 9,9]$, where 1.0001 is used to avoid $c_{i j}=1$ to prevent new logical error from being generated. Note that the constraint for $c_{i j}$ in (14) is for those elements greater than or equal to $1\left(c_{i j} \geqslant 1\right)$, as for $c_{i j}<1$, we just need to modify $c_{j i}\left(c_{j i}>1\right)$ firstly using (14), and then get $c_{i j}$ by $c_{i j}=1 / c_{j i}$.

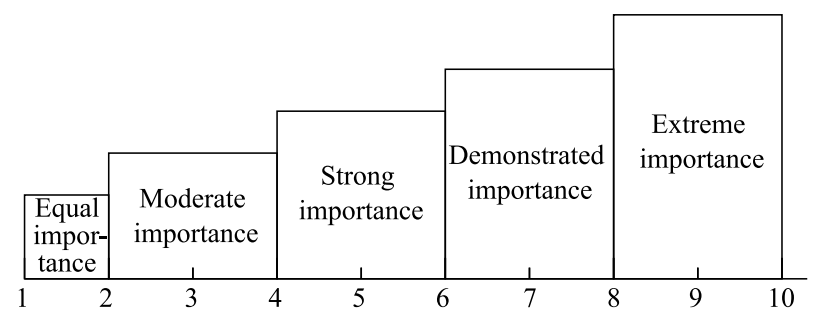

Fig. 7 Relationship between tolerance deviations interval and linguistic description

For a special case, if $\delta=0$, the model makes changes only to $x_{i}$, the elements in the 3 -node directed circuit.

(iii) Model 3: Our objective function is to minimize the total perturbation of the original PCM, and the constraints are the same as (14).

$$
\begin{gathered}
\min \left\{\varepsilon=\frac{1}{n} \sqrt{\left.\sum_{i=1}^{n} \sum_{j=1}^{n}\left(c_{i j}-a_{i j}\right)^{2}\right\}}\right\} \\
\text { s.t. } c_{i j} \in\left[\max \left\{1.0001, a_{i j}-\delta\right\}, \min \left\{a_{i j}+\delta, 9\right\}\right], \\
c_{i j} \notin\left\{x_{1}, x_{2}, \ldots, x_{m}\right\} ; c_{i j} \geqslant 1 \\
x_{i} \in[1 / 9,9], \forall i \in\{1,2, \ldots, m\} \\
\operatorname{CR}(\boldsymbol{C}) \leqslant 0.1
\end{gathered}
$$

where $\varepsilon$ denotes the total deviation extent between the modified PCM $\boldsymbol{C}$ and the original PCM $\boldsymbol{A}$, and $c_{i j}$ and $x_{i}$ have the same meaning as (14). Note that $c_{i j}$ denotes the logically consistent elements, the logically inconsistent elements $x_{i}(i=1,2, \ldots, m)$ are not considered in the calculation of total perturbation $\varepsilon$.

(iv) Model 4: This model is almost the same as Model 3 except the objective function.

$$
\min \left\{\sigma=\max \left\{\left|c_{i j}-a_{i j}\right|\right\}\right\}
$$

$$
\begin{gathered}
\text { s.t. } c_{i j} \in\left[\max \left\{1.0001, a_{i j}-\delta\right\}, \min \left\{a_{i j}+\delta, 9\right\}\right], \\
c_{i j} \notin\left\{x_{1}, x_{2}, \ldots, x_{m}\right\} ; c_{i j} \geqslant 1 \\
x_{i} \in[1 / 9,9], \forall i \in\{1,2, \ldots, m\} \\
\operatorname{CR}(\boldsymbol{C}) \leqslant 0.1
\end{gathered}
$$

where $\sigma$ denotes the maximum deviation among all modified elements that are logically consistent, similar to Model 3, the logically inconsistent elements $x_{i}(i=$ $1,2, \ldots, m)$ are not considered in the calculation of maximum deviation $\sigma$.

\subsubsection{Consistency improving strategy}

Since four models have been proposed in Section 3.2.1, we will focus on how to use these models to solve the inconsistency problems.

We will first use Model 1 and Model 2 to assure if it is feasible to realize the acceptable consistent level by only modifying the elements with logical error. For Model 1, it is required to specify which elements in the directed circuit have logical error so that we can redirect them to improve the logical inconsistency. As a result, we need to list all the possible modification combinations and solve each combination by the optimization model in (13). For Model 2, unlike Model 1, we do not need to specify which elements have logical errors, but consider all elements in the 3-node directed circuits as unknown, and substitute them with the best value on $[1 / 9,9]$ to improve the numerical consistency. Note that we keep the other elements unchanged here, that is, let $\delta=0$ in Model 2. If Model 1 or Model 2 (with $\delta=0$ ) has found acceptable solutions with logical consistency, which means that the acceptable consistent level is obtained without modifying any other elements except logically inconsistent ones, then the algorithm ends here; otherwise, we have to modify the other elements limitedly as well as those with logical error by using Model 3 and Model 4.

Model 3 and Model 4 are designed to realize acceptable consistent level by modifying all elements of the PCM, while preserving most information of the logically consistent elements. Theoretically, it is assured that Model 2 can find a best solution when $\delta$ is large enough. Because when $\delta$ is large enough, the consistency improving process is like searching for the optimum value of each element $a_{i j}$ within the whole scope of $[1 / 9,9]$, and the optimum solution with $\mathrm{CR}=0$ can be found without doubt. However, we hope $\delta$ to be within the acceptable range $\left(\delta \leqslant \delta_{\max }=1\right)$, such as 0.5 , since when an element is modified beyond \pm 0.5 , it may change the original judgment of the DM, e.g., changing $a_{i j}$ from 3 to 3.1 (i.e., $\delta=0.1$ ) may make no difference for the DM in linguistic importance in Table 1, but if changing it from 3 to 5 (here, $\delta=2$, which is beyond 
the tolerance deviations intervals in Table 1) may have altered the judgment of the DM. Hence, when all elements are modified within a reasonable range (e.g., $\delta=0.5$ ), it means the modified PCM by Model 3 and Model 4 is acceptable and feasible.

The process is shown in Fig. 8.

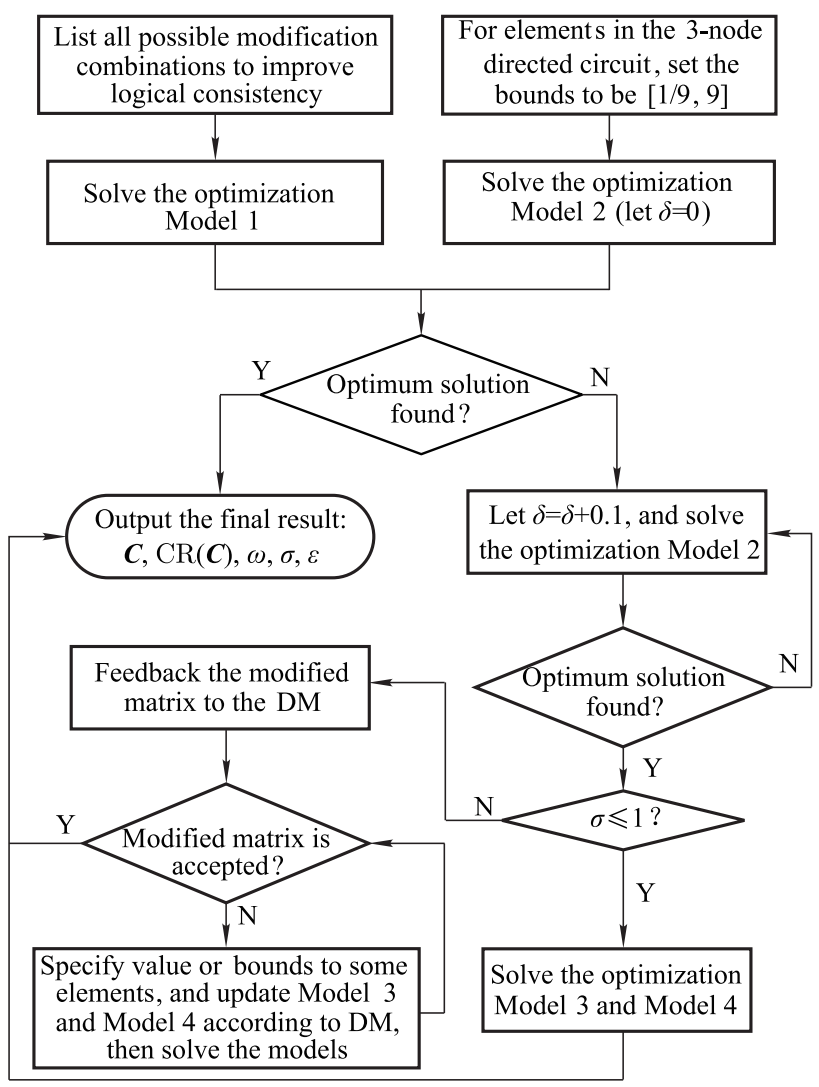

Fig. 8 Consistency improving process based on optimization models

Step 1 List all possible modification combinations to improve logical consistency. Firstly, find all 3-node directed circuits, and then redirect one or two elements using (13) in each circuit to obtain potential feasible modification combinations. Make such redirection to cover all possible combinations. If there are $q$ elements in all 3-node directed circuits, note that we only consider elements in the upper diagonal triangular matrix of the PCM, we will have

$\sum_{i=1}^{q-1} \mathrm{C}_{q}^{i}$ possible combinations, since $\mathrm{C}_{q}^{q}$ will not eliminate any directed circuit, but redirect the circuits. For example, if $q=3$, we can redirect any one or two element(s), which make $\mathrm{C}_{3}^{1}+\mathrm{C}_{3}^{2}=6$ possible combinations. Then, we will choose the possible modification combinations from these $\sum_{i=1}^{q-1} \mathrm{C}_{q}^{i}$ situations that can improve logical consistency by (2) and Corollary 1 (see Example 3 in Section 4 for more illustration).

Step 2 Generate the upper and lower bounds.

For elements with logical error in Model 1, according to $(13)$, if it is greater than 1 , set the bounds to $[1 / 9$, 0.999 9]; otherwise, set the bounds to [1.000 1, 9]. For other elements $a_{i j}$, we make no changes to them, thus the bounds are set to $\left[a_{i j}, a_{i j}\right]$.

For elements in the 3 -node directed circuit in Models 2, 3 , and 4 , set the bounds to $[1 / 9,9]$. For other elements $a_{i j}$, set the bounds as follows.

Let $\delta=0$, and the maximum deviation $\delta_{\max }=1$, i.e., $\delta \leqslant \delta_{\max }$. For $a_{i j}>1, i \neq j$, we have $a_{i j} \in\left[a_{i j}^{L}, a_{i j}^{U}\right]$.

$$
\left\{\begin{array}{l}
a_{i j}^{L}= \begin{cases}a_{i j}-\delta, & a_{i j}-\delta>1 \\
1.0001, & \text { otherwise }\end{cases} \\
a_{i j}^{U}= \begin{cases}a_{i j}+\delta, & a_{i j}+\delta<9 \\
9, & \text { otherwise }\end{cases}
\end{array}\right.
$$

where $a_{i j}^{L}$ denotes the lower bound, and $a_{i j}^{U}$ denotes the upper bound.

For each modified element $c_{i j}$, we have

$$
c_{i j} \in \begin{cases}{\left[a_{i j}^{L}, a_{i j}^{U}\right],} & a_{i j}>1 \\ {\left[\frac{1}{a_{j i}^{U}}, \frac{1}{a_{j i}^{L}}\right],} & \text { otherwise }\end{cases}
$$

where $a_{i j}^{L}$ and $a_{i j}^{U}$ are obtained by (17).

Step 3 Solve the optimization model. Using the MATLAB function fmincon or other global optimization algorithms, Model 1 and Model 2 with $\delta=0$ can be easily solved.

If the optimum solution exists, which means the numerical consistency is satisfied since $\mathrm{CR} \leqslant 0.1$ according to the constraints of (13) and (14), and also the logical consistency is satisfied since no directed circuits exist by Step 1, then we get the final modified PCM, go to Step 7. Otherwise, if no optimum solution is found, we have to modify logically consistent elements to reach numerical consistency by Model 2 . Let $\delta=\delta+0.1$, go to Step 2. Obviously, if $\delta$ is large enough, Model 2 can find the optimum solution, and if it does, we go to Step 4.

Step 4 Evaluate the optimum solution. If $\sigma \leqslant 1(\sigma$ is defined in (16)), accept the solution, and go to Step 6, which aims to realize the acceptable consistent level while preserving most of the logically consistent elements' information; otherwise, $\sigma>1$, which means the maximum deviation among modified elements is greater than $\delta_{\max }=1$, we think the modification deviation is so large that the original judgment may have been changed, go to Step 5 to ask for advice from the DM.

Step 5 Feedback the modified PCM to the DM. Since some modifications may have changed the original judgment, it is up to the DM to decide if the modifications are 
acceptable. If the modified PCM is accepted, go to Step 7; otherwise, ask the DM to give some advices so that more constraints are added to the models, e.g., re-specifying the value of some uncertain elements with relatively larger deviations, or specifying the feasible bounds to those elements that the DM is not so certain about, and go to Step 6 to use Model 3 and Model 4 to optimize. The process of optimizing, feedback and optimizing is repeated till an acceptable PCM is obtained, and then go to Step 7.

Step 6 Solve Model 3 and Model 4 by using the MATLAB function fmincon or other global optimization algo- rithms.

Step 7 Output the final result, including the improved PCM $\boldsymbol{C}$, consistent ratio $\mathrm{CR}(\boldsymbol{C})$, priority vector $\boldsymbol{\omega}$, as well as the indices of modification effectiveness $\sigma$ and $\varepsilon$ defined in (15) and (16).

\subsection{Iterative feedback procedure}

The following procedure contains the whole process including directed circuit analysis and the consistency improving method based on optimization, which is presented in Fig. 9.

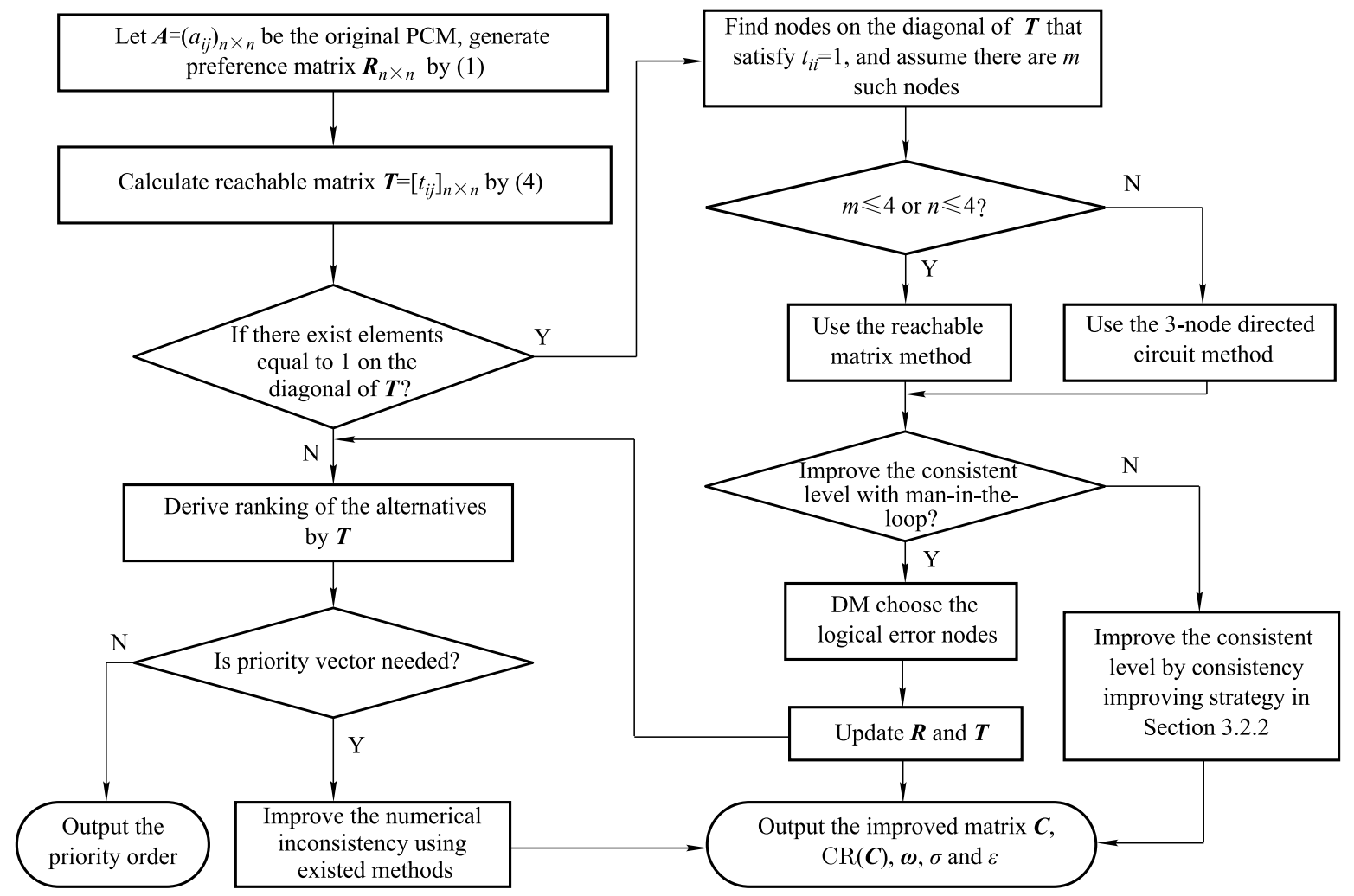

Fig. 9 The whole process of the consistency improving method based on directed circuit analysis

Step 1 Calculate reachable matrix $\boldsymbol{T}=\left[t_{i j}\right]_{n \times n}$ by (4).

Step 2 Analyze the logical consistency. If there exist elements equal to 1 on the main diagonal of reachable matrix $\boldsymbol{T}$, the matrix is logically inconsistent by Theorem 3 , go to Step 5; otherwise, go to Step 3.

Step 3 Derive ranking of the alternatives by $\boldsymbol{T}$ according to Theorem 5 and (5). If we only want to determine the priority order instead of the priority vector, and if each row has different $S_{i}$ in (5), the ranking of the alternatives is obtained, end the procedure; otherwise, go to Step 4.

Step 4 Improve numerical inconsistency. Since the matrix is logically consistent, we only need to improve numerical inconsistency. There are many studies to attain this goal such as [5], [6], and [26]. Finally, output the results: the improved PCM $\boldsymbol{C}, \mathrm{CR}(\boldsymbol{C}), \boldsymbol{\omega}, \sigma$ and $\varepsilon$.

Step 5 Find all 3-node directed circuits. Assume that there are $m$ nodes $\left\{i_{1}, i_{2}, \ldots, i_{m}\right\}$ on the main diagonal of $\boldsymbol{T}$ that satisfy $t_{i i}=1\left(i \in\left\{i_{1}, i_{2}, \ldots, i_{m}\right\}\right)$, we have $m \geqslant 3$ by Corollary 3 . To find all 3 -node directed circuits, if $m \leqslant 4$, use the reachable matrix method; otherwise, use the 3-node directed circuit method. Then, go to Step 6 if the DM is involved, or go to Step 7 if the DM is not involved.

Step 6 Improve the consistent level with man-in-theloop. When the DM is involved in modifying the PCM, the DM needs to check all the 3-node directed circuits to choose the logical error node(s) so that all logical inconsistencies are eliminated. For example, there is a 3-node 
directed circuit: $1 \rightarrow 2 \rightarrow 3 \rightarrow 1$, the DM finds out alternative 1 is preferred to alternative 3 , then we set $a_{13}>1$, as such, this logical inconsistency is eliminated. Update $\boldsymbol{R}$ and $\boldsymbol{T}$, go to Step 4. Though involving DM is effective and accurate, in some cases, there are too many 3-node directed circuits and crossover edges, which may confuse the DM. For such situation, we go to Step 7.

Step 7 Improve the consistent level without man-inthe-loop. We adopt the consistency improving strategy in Section 3.2.2. If the improved PCM is acceptable in both logical and numerical consistency, output the results.

\section{Illustrative examples}

Since the situation with man-in-the-loop is much easier than the situation without man-in-the-loop, and both situations share similar processes, we will only discuss the situation without man-in-the-loop.

Example 1 The $8 \times 8 \mathrm{PCM} \boldsymbol{A}$, which is also analyzed in [5-7] and [27], is inconsistent with $\mathrm{CR}=0.1691>0.1$.

\begin{tabular}{c|cccccccc|} 
& 1 & 2 & 3 & 4 & 5 & 6 & 7 & 8 \\
\hline 1 & 1 & 5 & 3 & 7 & 6 & 6 & $1 / 3$ & $1 / 4$ \\
2 & $1 / 5$ & 1 & $1 / 3$ & 5 & 3 & 3 & $1 / 5$ & $1 / 7$ \\
3 & $1 / 3$ & 3 & 1 & 6 & 3 & 4 & 6 & $1 / 5$ \\
4 & $1 / 7$ & $1 / 5$ & $1 / 6$ & 1 & $1 / 3$ & $1 / 4$ & $1 / 7$ & $1 / 8$ \\
5 & $1 / 6$ & $1 / 3$ & $1 / 3$ & 3 & 1 & $1 / 2$ & $1 / 5$ & $1 / 6$ \\
6 & $1 / 6$ & $1 / 3$ & $1 / 4$ & 4 & 2 & 1 & $1 / 5$ & $1 / 6$ \\
7 & 3 & 5 & $1 / 6$ & 7 & 5 & 5 & 1 & $1 / 2$ \\
8 & 4 & 7 & 5 & 8 & 6 & 6 & 2 & 1
\end{tabular}

(i) Analyze the logical consistency. By (1) and (4), we have

$$
\begin{aligned}
\boldsymbol{R} & =\left[\begin{array}{llllllll}
0 & 1 & 1 & 1 & 1 & 1 & 0 & 0 \\
0 & 0 & 0 & 1 & 1 & 1 & 0 & 0 \\
0 & 1 & 0 & 1 & 1 & 1 & 1 & 0 \\
0 & 0 & 0 & 0 & 0 & 0 & 0 & 0 \\
0 & 0 & 0 & 1 & 0 & 0 & 0 & 0 \\
0 & 0 & 0 & 1 & 1 & 0 & 0 & 0 \\
1 & 1 & 0 & 1 & 1 & 1 & 0 & 0 \\
1 & 1 & 1 & 1 & 1 & 1 & 1 & 0
\end{array}\right] \\
\boldsymbol{T} & =\left[\begin{array}{llllllll}
1 & 1 & 1 & 1 & 1 & 1 & 1 & 0 \\
0 & 0 & 0 & 1 & 1 & 1 & 0 & 0 \\
1 & 1 & 1 & 1 & 1 & 1 & 1 & 0 \\
0 & 0 & 0 & 0 & 0 & 0 & 0 & 0 \\
0 & 0 & 0 & 1 & 0 & 0 & 0 & 0 \\
0 & 0 & 0 & 1 & 1 & 0 & 0 & 0 \\
1 & 1 & 1 & 1 & 1 & 1 & 1 & 0 \\
1 & 1 & 1 & 1 & 1 & 1 & 1 & 0
\end{array}\right] .
\end{aligned}
$$

Obviously, there exist three elements equal to 1 on the main diagonal of reachable matrix $\boldsymbol{T}, t_{11}=1, t_{33}=1$, $t_{77}=1$, and $m=3$. By Theorem 4 and the reachable matrix method in Section 3.1, we get a 3-node directed circuit: $1 \rightarrow 3 \rightarrow 7 \rightarrow 1$. Meanwhile, we can also find that the number of minimum directed circuits (or 3-node directed circuits) $c=1$ by (2), which is consistent with the reachable matrix method.

(ii) Improve the consistency level. By the consistency improving strategy in Section 3.2.2, we first use Model 1, and list all the possible modification combinations as follows.

Situation 1 Suppose there is one element that has logical error in the 3-node directed circuit, we have three possible modification combinations, $\left\{a_{13}\right\},\left\{a_{17}\right\},\left\{a_{37}\right\}$, which means changing the element in the bracket by (13) can eliminate the directed circuit, thus make the PCM logically consistent.

Situation 2 Suppose there are two elements that have logical errors, we have three possible modification combinations, $\left\{a_{13}, a_{17}\right\},\left\{a_{17}, a_{37}\right\},\left\{a_{13}, a_{37}\right\}$.

By using Model 1 for each combination, the results are listed in Table 2. We can conclude that when the logical error belongs to these situations: $\left\{a_{37}\right\},\left\{a_{17}, a_{37}\right\},\left\{a_{13}\right.$, $\left.a_{37}\right\}$, the acceptable consistent level can be reached.

Comparisons among some established methods and our method are given in Table 3. Considering $\left\{a_{37}\right\}$ has logical error, it will be modified to 0.4645 according to Table 2, which is almost the same as [27] and [7] where $a_{37}$ is modified to 0.5 , however, our method is more numerically consistent with smaller CR (0.082 19) than [27] and [7]'s 0.082 24. For [5] and [6], the improved PCMs are numerically consistent, but logically inconsistent since the directed circuit $1 \rightarrow 3 \rightarrow 7 \rightarrow 1$ still exists in the improved PCM. Obviously, our result is much better than these studies.

Similarly, we can also use Model 2 to solve the problem where $\delta$ is set to be 0 and 0.5 respectively, and we obtain Situation 3.

Situation 3 Suppose three elements in the 3-node directed circuit are unknown, that is, we assume $\left\{a_{13}, a_{17}\right.$, $\left.a_{37}\right\}$ are missing, thus, we set the bounds of these elements to be $[1 / 9,9]$. Using Model 2, the results are shown in Table 2 . It can be seen that the improved PCM has reached acceptable consistent level by just modifying logically inconsistent elements without changing other elements $(\delta=0)$, thus $\varepsilon=0$ and $\sigma=0$ by (15) and (16). As a result, we will not consider the situation $\delta=0.5$ since it is unnecessary to modify other elements which can only help to make the two indices $\varepsilon$ and $\sigma$ greater than 0 . However, we have also solved the situation $\delta=0.5$ and presented the results in Table 2 and Table 4 for comparison. Comparing the two situations: Model 2 with $\delta=0.5$ and $\delta=0$, the former situation can obtain better consistent ratio $(\mathrm{CR}=0.0378)$ but preserve less information than the latter situation, that is, the two indices $\varepsilon$ and $\sigma$ are greater than 0 (the situation $\delta=0)$. 
Table 2 Output results for different situations in Example 1

\begin{tabular}{|c|c|c|c|c|c|c|}
\hline \multirow[b]{2}{*}{ Situation } & \multirow{2}{*}{$\begin{array}{l}\text { Element with } \\
\text { logical error }\end{array}$} & \multicolumn{5}{|c|}{ Modified matrix } \\
\hline & & $\begin{array}{l}\text { Modified } \\
\text { element }\end{array}$ & $\begin{array}{c}\text { Logical } \\
\text { consistency }\end{array}$ & CR & $\begin{array}{c}\text { Consistent } \\
\text { level }\end{array}$ & $\begin{array}{l}\text { Priority vector } \boldsymbol{\omega} \text { and } \\
\text { priority order }\end{array}$ \\
\hline \multirow{2}{*}{$\begin{array}{l}\text { Situation } 1 \\
\text { (Assume one } \\
\text { element has } \\
\text { logical error) }\end{array}$} & $\begin{array}{l}a_{13} \text { redirect, and other ele- } \\
\text { ments keep unchanged }\end{array}$ & $a_{13}=0.7202$ & Acceptable & 0.1433 & $\begin{array}{c}\text { Not } \\
\text { acceptable }\end{array}$ & - \\
\hline & $\begin{array}{l}a_{17} \text { redirect, and other ele- } \\
\text { ments keep unchanged }\end{array}$ & $a_{17}=1.762$ & Acceptable & 0.1342 & $\begin{array}{c}\text { Not } \\
\text { acceptable }\end{array}$ & - \\
\hline \multirow{3}{*}{$\begin{array}{c}\text { Situation } 2 \\
\text { (Assume two } \\
\text { elements have } \\
\text { logical error) }\end{array}$} & $\begin{array}{l}a_{13} \text { and } a_{17} \text { redirect, and } \\
\text { other elements keep un- } \\
\text { changed }\end{array}$ & $\begin{array}{l}a_{13}=0.9239 \\
a_{17}=1.3625\end{array}$ & Acceptable & 0.1214 & $\begin{array}{c}\text { Not } \\
\text { acceptable }\end{array}$ & - \\
\hline & $\begin{array}{l}a_{17} \text { and } a_{37} \text { redirect, and } \\
\text { other elements keep un- } \\
\text { changed }\end{array}$ & $\begin{array}{l}a_{17}=1.0759 \\
a_{37}=0.5752\end{array}$ & Acceptable & 0.0703 & Acceptable & $\begin{array}{c}\{0.1972,0.0636,0.1067,0.0196, \\
0.0344,0.042,0.18,0.3565\} \\
8 \succ 1 \succ 7 \succ 3 \succ 2 \succ 6 \succ 5 \succ 4\end{array}$ \\
\hline & $\begin{array}{l}a_{13} \text { and } a_{37} \text { redirect, and } \\
\text { other elements keep un- } \\
\text { changed }\end{array}$ & $\begin{array}{l}a_{13}=0.9999 \\
a_{37}=0.5611\end{array}$ & Acceptable & 0.0759 & Acceptable & $\begin{array}{c}\{0.1539,0.0634,0.1194,0.0195, \\
0.0346,0.042,0.2176,0.3494\} \\
8 \succ 7 \succ 1 \succ 3 \succ 2 \succ 6 \succ 5 \succ 4\end{array}$ \\
\hline \multirow{2}{*}{$\begin{array}{c}\text { Situation } 3 \\
\text { (Assume elements } \\
\text { in the 3-node } \\
\text { directed circuit } \\
\text { as unknown) }\end{array}$} & $\begin{array}{l}\text { Consider } a_{13}, \quad a_{17} \text { and } \\
a_{37} \text { as unknown }(\delta=0)\end{array}$ & $\begin{array}{l}a_{13}=1.4763 \\
a_{17}=0.9271 \\
a_{37}=0.6264\end{array}$ & Acceptable & 0.0656 & Acceptable & $\begin{array}{c}\{0.178,0.0647,0.1172,0.0198, \\
0.035,0.0427,0.1842,0.3586\} \\
8 \succ 7 \succ 1 \succ 3 \succ 2 \succ 6 \succ 5 \succ 4\end{array}$ \\
\hline & $\begin{array}{l}\text { Consider } a_{13}, a_{17} \text { and } \\
a_{37} \text { as unknown }(\delta= \\
0.5)\end{array}$ & see Table 4 & Acceptable & 0.0378 & Acceptable & $\begin{array}{c}\{0.1776,0.0634,0.1177,0.0203, \\
0.0352,0.0419,0.1966,0.3474\} \\
8 \succ 7 \succ 1 \succ 3 \succ 2 \succ 6 \succ 5 \succ 4\end{array}$ \\
\hline
\end{tabular}

Table 3 Comparisons among some established methods and our method

\begin{tabular}{|c|c|c|c|c|c|}
\hline \multirow{2}{*}{ Method } & \multicolumn{5}{|c|}{ Modified matrix } \\
\hline & Modified element & Logical consistency & $\mathrm{CR}$ & Consistent level & $\varepsilon$ \\
\hline$[5]$ & $\begin{array}{l}\text { All elements are modified with maximum } \\
\text { modification } \sigma=1.845\end{array}$ & $\begin{array}{l}\text { Not acceptable with directed } \\
\quad \text { circuit } 1 \rightarrow 3 \rightarrow 7 \rightarrow 1\end{array}$ & 0.097 & Acceptable & 0.589 \\
\hline [6] & $\begin{array}{l}\text { All elements are modified with maximum } \\
\text { modification } \sigma=1.713\end{array}$ & $\begin{array}{l}\text { Not acceptable with directed } \\
\quad \text { circuit } 1 \rightarrow 3 \rightarrow 7 \rightarrow 1\end{array}$ & 0.0997 & Acceptable & 0.448 \\
\hline [27] and [7] & $a_{37}$ is modified from 6 to 0.5 & Acceptable & 0.08224 & Acceptable & 0 \\
\hline
\end{tabular}

Table 4 Modified elements for Situation $3(\delta=0.5)$ (Elements in the directed circuit are in bold)

\begin{tabular}{|c|c|c|c|c|c|c|c|}
\hline Element & $\begin{array}{c}\text { Original } \\
\text { value }\end{array}$ & $\begin{array}{c}\text { Modification } \\
\text { bound }\end{array}$ & $\begin{array}{l}\text { Modified } \\
\text { value }\end{array}$ & Element & $\begin{array}{c}\text { Original } \\
\text { value }\end{array}$ & $\begin{array}{c}\text { Modification } \\
\text { bound }\end{array}$ & $\begin{array}{c}\text { Modified } \\
\text { value }\end{array}$ \\
\hline$a_{12}$ & 5 & {$[4.5,5.5]$} & 4.5 & $a_{35}$ & 3 & {$[2.5,3.5]$} & 3.3266 \\
\hline$a_{13}$ & 3 & {$[1 / 9,9]$} & 1.4257 & $a_{36}$ & 4 & {$[3.5,4.5]$} & 3.5 \\
\hline$a_{14}$ & 7 & {$[6.5,7.5]$} & 7.4961 & $a_{37}$ & 6 & {$[1 / 9,9]$} & 0.5904 \\
\hline$a_{15}$ & 6 & {$[5.5,6.5]$} & 5.778 & $a_{38}$ & 0.2 & {$[1 / 5.5,1 / 4.5]$} & 0.2222 \\
\hline$a_{16}$ & 6 & {$[5.5,6.5]$} & 5.5 & $a_{45}$ & 0.3333 & {$[1 / 3.5,1 / 2.5]$} & 0.4 \\
\hline$a_{17}$ & 0.3333 & {$[1 / 9,9]$} & 0.8691 & $a_{46}$ & 0.25 & {$[1 / 4.5,1 / 3.5]$} & 0.2857 \\
\hline$a_{18}$ & 0.25 & {$[1 / 4.5,1 / 3.5]$} & 0.2857 & $a_{47}$ & 0.1429 & {$[1 / 7.5,1 / 6.5]$} & 0.1333 \\
\hline$a_{23}$ & 0.3333 & {$[1 / 3.5,1 / 2.5]$} & 0.4 & $a_{48}$ & 0.125 & {$[1 / 8.5,1 / 7.5]$} & 0.1176 \\
\hline$a_{24}$ & 5 & {$[4.5,5.5]$} & 4.5 & $a_{56}$ & 0.5 & {$[1 / 2.5,1 / 1.5]$} & 0.6667 \\
\hline$a_{25}$ & 3 & {$[2.5,3.5]$} & 2.5 & $a_{57}$ & 0.2 & {$[1 / 5.5,1 / 4.5]$} & 0.1818 \\
\hline$a_{26}$ & 3 & {$[2.5,3.5]$} & 2.5 & $a_{58}$ & 0.1667 & {$[1 / 6.5,1 / 5.5]$} & 0.1538 \\
\hline$a_{27}$ & 0.2 & {$[1 / 5.5,1 / 4.5]$} & 0.2222 & $a_{67}$ & 0.2 & {$[1 / 5.5,1 / 4.5]$} & 0.2055 \\
\hline$a_{28}$ & 0.1429 & {$[1 / 7.5,1 / 6.5]$} & 0.1538 & $a_{68}$ & 0.1667 & {$[1 / 6.5,1 / 5.5]$} & 0.1538 \\
\hline$a_{34}$ & 6 & {$[5.5,6.5]$} & 6.2784 & $a_{78}$ & 0.5 & {$[1 / 2.5,1 / 1.5]$} & 0.6065 \\
\hline
\end{tabular}


Example 2 The $7 \times 7$ PCM $\boldsymbol{A}$, which is also analyzed in [28], is inconsistent with $\mathrm{CR}=0.3061>0.1$.

\begin{tabular}{c|ccccccc|} 
& 1 & 2 & 3 & 4 & 5 & 6 & 7 \\
\hline 1 & 1 & 7 & 3 & 5 & 9 & 3 & 5 \\
2 & $1 / 7$ & 1 & 3 & 3 & 5 & 3 & 3 \\
3 & $1 / 3$ & $1 / 3$ & 1 & $1 / 5$ & $1 / 3$ & 3 & 3 \\
4 & $1 / 5$ & $1 / 3$ & 5 & 1 & 9 & 3 & $1 / 3$ \\
5 & $1 / 9$ & $1 / 5$ & 3 & $1 / 9$ & 1 & 3 & $1 / 5$ \\
6 & $1 / 3$ & $1 / 3$ & $1 / 3$ & $1 / 3$ & $1 / 3$ & 1 & $1 / 3$ \\
7 & $1 / 5$ & $1 / 3$ & $1 / 3$ & 3 & 5 & 3 & 1
\end{tabular}

(i) Analyze the logical consistency. By (1) and (4), we have

$$
\begin{aligned}
\boldsymbol{R} & =\left[\begin{array}{lllllll}
0 & 1 & 1 & 1 & 1 & 1 & 1 \\
0 & 0 & 1 & 1 & 1 & 1 & 1 \\
0 & 0 & 0 & 0 & 0 & 1 & 1 \\
0 & 0 & 1 & 0 & 1 & 1 & 0 \\
0 & 0 & 1 & 0 & 0 & 1 & 0 \\
0 & 0 & 0 & 0 & 0 & 0 & 0 \\
0 & 0 & 0 & 1 & 1 & 1 & 0
\end{array}\right] \\
\boldsymbol{T} & =\left[\begin{array}{lllllll}
0 & 1 & 1 & 1 & 1 & 1 & 1 \\
0 & 0 & 1 & 1 & 1 & 1 & 1 \\
0 & 0 & 1 & 1 & 1 & 1 & 1 \\
0 & 0 & 1 & 1 & 1 & 1 & 1 \\
0 & 0 & 1 & 1 & 1 & 1 & 1 \\
0 & 0 & 0 & 0 & 0 & 0 & 0 \\
0 & 0 & 1 & 1 & 1 & 1 & 1
\end{array}\right] .
\end{aligned}
$$

There exist four elements equal to 1 on the main diagonal of $\boldsymbol{T}$, i.e., $t_{33}=1, t_{44}=1, t_{55}=1, t_{77}=1$, and $m=4$. And by (2), the number of minimum directed circuits $c=2$, thus we can conclude that there exist two 3node directed circuits in $\boldsymbol{R}$. To illustrate our methods, we will determine all the 3-node directed circuits by the reachable matrix method and the 3 -node directed circuit method respectively.

(i) Reachable matrix method

There are four nodes $\{3,4,5,7\}$ on the 3-node directed circuits, and we need to analyze $\mathrm{C}_{4}^{3}=4$ possible 3-node directed circuits.

Case 1 Nodes $\{3,4,5\}$. Since $a_{34}=1 / 5, a_{35}=1 / 3$, we have $a_{3} \stackrel{5}{\prec} a_{4}, a_{3} \stackrel{3}{\prec} a_{5}$, we conclude that $a_{5} \prec a_{4}$ since $a_{4}$ is five times better than $a_{3}$, while $a_{5}$ is three times better than $a_{3}$, which means $a_{4}$ is more preferable. As $a_{45}=9$, which means $a_{4} \succ a_{5}$, the nodes $\{3,4,5\}$ cannot make a directed circuit.

Case 2 Nodes $\{3,4,7\}$. Since $a_{34}=1 / 5, a_{37}=3$. We have $a_{3} \stackrel{5}{\prec} a_{4}, a_{3} \succ a_{7}$. We conclude that $a_{7} \prec a_{4}$, but $a_{47}=1 / 3$, which means $a_{4} \prec a_{7}$, thus the nodes make a 3-node directed circuit: $7 \rightarrow 4 \rightarrow 3 \rightarrow 7$.

Case 3 Nodes $\{3,5,7\}$. Since $a_{35}=1 / 3, a_{37}=3$. We have $a_{3} \stackrel{3}{\prec} a_{5}, a_{3} \succ a_{7}$. We conclude that $a_{7} \prec a_{5}$, but $a_{57}=1 / 5$, which means $a_{5} \prec a_{7}$, thus the nodes make a 3-node directed circuit: $7 \rightarrow 5 \rightarrow 3 \rightarrow 7$.

Case 4 Nodes $\{4,5,7\}$. Since $a_{45}=9, a_{47}=1 / 3$, we have $a_{4} \stackrel{9}{\succ} a_{5}, a_{4} \stackrel{3}{\prec} a_{7}$, we conclude that $a_{5} \prec a_{7}$. As $a_{57}=1 / 5$, which means $a_{5} \prec a_{7}$, the nodes $\{4,5,7\}$ cannot make a directed circuit.

All in all, we have found two 3-node directed circuits: $7 \rightarrow 4 \rightarrow 3 \rightarrow 7,7 \rightarrow 5 \rightarrow 3 \rightarrow 7$.

(ii) 3-node directed circuit method

Calculate the edge matrix $\boldsymbol{E}(1)$ and the path matrix $\boldsymbol{W}(1)$ by (6) and (7).

$$
\boldsymbol{E}(1)=\left[\begin{array}{ccccccc}
\phi & \left\{v_{1} v_{2}\right\} & \left\{v_{1} v_{3}\right\} & \left\{v_{1} v_{4}\right\} & \left\{v_{1} v_{5}\right\} & \left\{v_{1} v_{6}\right\} & \left\{v_{1} v_{7}\right\} \\
\phi & \phi & \left\{v_{2} v_{3}\right\} & \left\{v_{2} v_{4}\right\} & \left\{v_{2} v_{5}\right\} & \left\{v_{2} v_{6}\right\} & \left\{v_{2} v_{7}\right\} \\
\phi & \phi & \phi & \phi & \phi & \left\{v_{3} v_{6}\right\} & \left\{v_{3} v_{7}\right\} \\
\phi & \phi & \left\{v_{4} v_{3}\right\} & \phi & \left\{v_{4} v_{5}\right\} & \left\{v_{4} v_{6}\right\} & \phi \\
\phi & \phi & \left\{v_{5} v_{3}\right\} & \phi & \phi & \left\{v_{5} v_{6}\right\} & \phi \\
\phi & \phi & \phi & \phi & \phi & \phi & \phi \\
\phi & \phi & \phi & \left\{v_{7} v_{4}\right\} & \left\{v_{7} v_{5}\right\} & \left\{v_{7} v_{6}\right\} & \phi
\end{array}\right], \quad \boldsymbol{W}(1)=\left[\begin{array}{cccccc}
\phi & \phi & \phi & \phi & \phi & \phi \\
\phi & \phi & \phi & \phi & \phi & \phi \\
\phi & \phi & \phi & \phi & \phi & \phi \\
\phi & \phi & \phi & \phi & \phi & \phi \\
\phi & \phi & \phi & \phi & \phi & \phi \\
\phi & \phi & \phi & \phi & \phi & \phi
\end{array}\right]
$$

Let $k=2$, calculate $\boldsymbol{E}(2)$ by (10) and (11), and then we obtain $\boldsymbol{W}(2)$ by (7).

$$
\boldsymbol{E}(2)=\left[\begin{array}{cccccc}
\phi & \left\{v_{2} v_{3}\right\} & \left\{v_{2} v_{4}\right\} & \left\{v_{2} v_{5}\right\} & \left\{v_{2} v_{6}\right\} & \left\{v_{2} v_{7}\right\} \\
\phi & \phi & \phi & \phi & \left\{v_{3} v_{6}\right\} & \left\{v_{3} v_{7}\right\} \\
\phi & \left\{v_{4} v_{3}\right\} & \phi & \left\{v_{4} v_{5}\right\} & \left\{v_{4} v_{6}\right\} & \phi \\
\phi & \left\{v_{5} v_{3}\right\} & \phi & \phi & \left\{v_{5} v_{6}\right\} & \phi \\
\phi & \phi & \phi & \phi & \phi & \phi \\
\phi & \phi & \left\{v_{7} v_{4}\right\} & \left\{v_{7} v_{5}\right\} & \left\{v_{7} v_{6}\right\} & \phi
\end{array}\right], \quad \boldsymbol{W}(2)=\left[\begin{array}{ccccc}
\phi & \phi & \phi & \phi & \phi \\
\phi & \phi & \phi & \phi & \phi \\
\phi & \phi & \phi & \phi & \phi \\
\phi & \phi & \phi & \phi & \phi \\
\phi & \phi & \phi & \phi & \phi
\end{array}\right]
$$

Let $k=k+1$, continue to calculate $\boldsymbol{E}(3), \boldsymbol{W}(3), \boldsymbol{E}(4), \boldsymbol{W}(4)$ : 


$$
\begin{aligned}
& \boldsymbol{E}(3)=\left[\begin{array}{ccccc}
\phi & \phi & \phi & \left\{v_{3} v_{6}\right\} & \left\{v_{3} v_{7}\right\} \\
\left\{v_{4} v_{3}\right\} & \phi & \left\{v_{4} v_{5}\right\} & \left\{v_{4} v_{6}\right\} & \phi \\
\left\{v_{5} v_{3}\right\} & \phi & \phi & \left\{v_{5} v_{6}\right\} & \phi \\
\phi & \phi & \phi & \phi & \phi \\
\phi & \left\{v_{7} v_{4}\right\} & \left\{v_{7} v_{5}\right\} & \left\{v_{7} v_{6}\right\} & \phi
\end{array}\right], \quad \boldsymbol{W}(3)=\left[\begin{array}{cccc}
\phi & \phi & \left\{v_{4} v_{3} v_{6}\right\} & \left\{v_{4} v_{3} v_{7}\right\} \\
\phi & \phi & \left\{v_{5} v_{3} v_{6}\right\} & \left\{v_{5} v_{3} v_{7}\right\} \\
\phi & \phi & \phi & \phi \\
\phi & \phi & \phi & \phi
\end{array}\right] \\
& \boldsymbol{E}(4)=\left[\begin{array}{cccc}
\phi & \left\{v_{4} v_{5}\right\} & \left\{v_{4} v_{3} v_{6}, v_{4} v_{6}\right\} & \left\{v_{4} v_{3} v_{7}\right\} \\
\phi & \phi & \left\{v_{5} v_{3} v_{6}, v_{5} v_{6}\right\} & \left\{v_{5} v_{3} v_{7}\right\} \\
\phi & \phi & \phi & \phi \\
\left\{v_{7} v_{4}\right\} & \left\{v_{7} v_{5}\right\} & \left\{v_{7} v_{6}\right\} & \phi
\end{array}\right] \\
& \boldsymbol{W}(4)=\left[\begin{array}{ccc}
\phi & \phi & \phi \\
\phi & \phi & \phi \\
\left\{v_{7} v_{4} v_{5}\right\} & \left\{v_{7} v_{4} v_{3} v_{6}, v_{7} v_{4} v_{6}\right\} & \left\{v_{7} v_{4} v_{3} v_{7}\right\}
\end{array}\right] \text {. }
\end{aligned}
$$

Since there exist non-empty elements $v_{7} v_{4} v_{3} v_{7}$ on the main diagonal of $\boldsymbol{W}(4)$, we obtain a directed circuit $v_{7} v_{4} v_{3} v_{7}$

Let $k=k+1$, continue to calculate $\boldsymbol{E}(5), \boldsymbol{W}(5)$ :

$$
\begin{aligned}
& \boldsymbol{E}(5)=\left[\begin{array}{ccc}
\phi & \left\{v_{5} v_{3} v_{6}, v_{5} v_{6}\right\} & \left\{v_{5} v_{3} v_{7}\right\} \\
\phi & \phi & \phi \\
\left\{v_{7} v_{4} v_{5}, v_{7} v_{5}\right\} & \left\{v_{7} v_{4} v_{3} v_{6}, v_{7} v_{4} v_{6}, v_{7} v_{6}\right\} & \left\{v_{7} v_{4} v_{3} v_{7}\right\}
\end{array}\right] \\
& \boldsymbol{W}(5)=\left[\begin{array}{cc}
\phi & \phi \\
\left\{v_{7} v_{4} v_{5} v_{3} v_{6}, v_{7} v_{4} v_{5} v_{6}, v_{7} v_{5} v_{3} v_{6}, v_{7} v_{5} v_{6}\right\} & \left\{v_{7} v_{4} v_{5} v_{3} v_{7}, v_{7} v_{5} v_{3} v_{7}\right\}
\end{array}\right] .
\end{aligned}
$$

We find non-empty elements $v_{7} v_{4} v_{5} v_{3} v_{7}$ and $v_{7} v_{5} v_{3} v_{7}$ on the main diagonal of $\boldsymbol{W}(5)$, thus two directed circuits are obtained, $v_{7} v_{4} v_{5} v_{3} v_{7}, v_{7} v_{5} v_{3} v_{7}$.
Let $k=k+1$, continue to calculate $\boldsymbol{E}(6), \boldsymbol{W}(6)$. As $k=7-1=6$ is satisfied, the process ends.

$$
\boldsymbol{E}(6)=\left[\begin{array}{cc}
\phi & \phi \\
\left\{v_{7} v_{4} v_{3} v_{6}, v_{7} v_{4} v_{6}, v_{7} v_{6}, v_{7} v_{4} v_{5} v_{3} v_{6},\right. & \left\{v_{7} v_{4} v_{5} v_{3} v_{7}, v_{7} v_{5} v_{3} v_{7}, v_{7} v_{4} v_{3} v_{7}\right\} \\
\left.v_{7} v_{4} v_{5} v_{6}, v_{7} v_{5} v_{3} v_{6}, v_{7} v_{5} v_{6}\right\} &
\end{array}\right]
$$

$$
\boldsymbol{W}(6)=[\phi]
$$$$
\boldsymbol{E}(7)=\left[\left\{v_{7} v_{4} v_{5} v_{3} v_{7}, v_{7} v_{5} v_{3} v_{7}, v_{7} v_{4} v_{3} v_{7}\right\}\right]
$$

In general, we obtain three directed circuits including $v_{7} v_{4} v_{3} v_{7}, v_{7} v_{4} v_{5} v_{3} v_{7}, v_{7} v_{5} v_{3} v_{7}$. And we select all the 3node directed circuits from them, and obtain: $7 \rightarrow 4 \rightarrow$ $3 \rightarrow 7,7 \rightarrow 5 \rightarrow 3 \rightarrow 7$.

(ii) Improve the consistency level. We list all the possible modification combinations as follows.

We firstly find all the possible elements of PCM from the 3-node directed circuits: $7 \rightarrow 4 \rightarrow 3 \rightarrow 7$, $7 \rightarrow 5 \rightarrow 3 \rightarrow 7$. There are five such elements, $a_{34}$, $a_{35}, a_{37}, a_{47}, a_{57}$. According to Section 3.2.2, there are $\mathrm{C}_{5}^{1}+\mathrm{C}_{5}^{2}+\mathrm{C}_{5}^{3}+\mathrm{C}_{5}^{4}=30$ possible combinations, among which only 12 combinations can eliminate all the directed circuits in that $c=0$ by (2) and Corollary 1, which we list as follows.

Situation 1 Suppose there is one element that has logical error, we should only assume $\left\{a_{37}\right\}$ to be the element with logic error so that the two directed circuits can be dismantled at the same time.

Situation 2 Suppose there are two elements that have logical error, then we have the following possible modification combinations, $\left\{a_{47}, a_{37}\right\},\left\{a_{35}, a_{37}\right\},\left\{a_{34}, a_{35}\right\}$, $\left\{a_{47}, a_{35}\right\},\left\{a_{47}, a_{57}\right\}$.

Situation 3 Suppose there are three elements that have logical error, then we have the following possible modification combinations, $\left\{a_{34}, a_{37}, a_{35}\right\},\left\{a_{47}, a_{37}, a_{35}\right\}$, $\left\{a_{47}, a_{34}, a_{35}\right\},\left\{a_{47}, a_{57}, a_{35}\right\},\left\{a_{47}, a_{37}, a_{57}\right\}$.

Situation 4 Suppose there are four elements that have logical error, then we have one possible modification combinations, $\left\{a_{34}, a_{47}, a_{35}, a_{57}\right\}$.

By using Model 1 for each combination, the results are listed in Table 5. Similarly, we can also use Model 2 to solve the problem where $\delta$ are set to 0 and 0.5 respectively, and we obtain Situation 5. 
WU Shihui et al.: A consistency improving method in the analytic hierarchy process based on directed circuit analysis

Table 5 Output results for different situations in Example 2

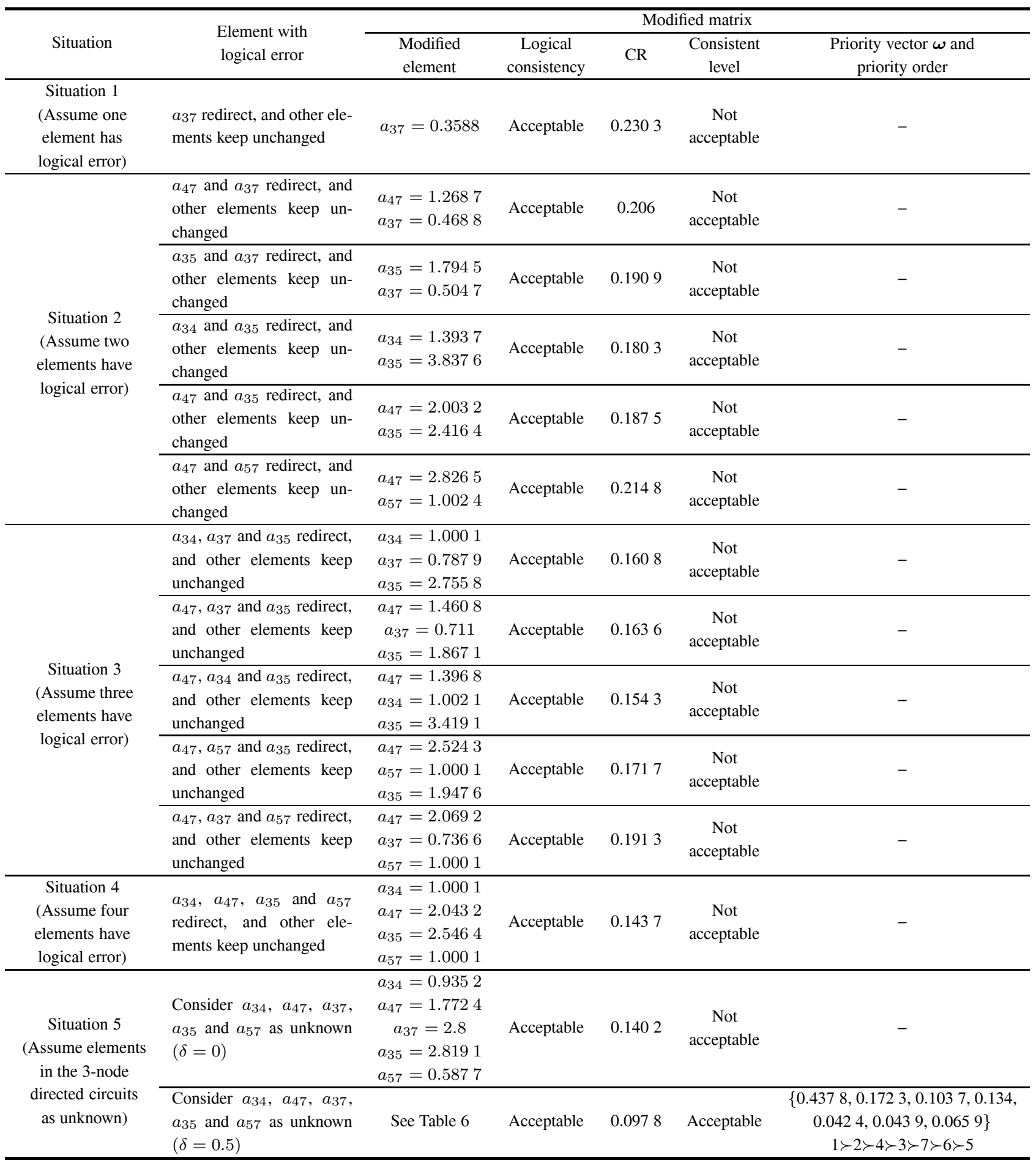

Situation 5 Suppose the five elements in the 3-node directed circuits, $a_{34}, a_{35}, a_{37}, a_{47}, a_{57}$ are unknown, we should set the bounds of these elements to be [1/9, 9]. Using Model 2 with $\delta=0$, the results are shown in Table 5. It can be seen that the improved PCM cannot reach the acceptable consistent level for $\delta=0$, which means it is not enough by just modifying the elements with logical error. And we continue to solve the situation using Model 2 with $\delta=0.5$, and the improved PCM is acceptable with $\mathrm{CR}=0.097$ 8. The results are shown in Table 5 and Table 6. Though the improved PCM obtained by Model 2 with $\delta=0.5$ is acceptable, we will use this as the initial so- 
lution to seek for a better PCM which can preserve most of the logically consistent elements' information by optimization models, Model 3 and Model 4.

(iii) Realize the acceptable consistent level while preserving most of the logically consistent elements' information.
Using Model 3 and Model 4, and set the modified elements of Situation $5(\delta=0.5)$ in Table 6 as the initial solution, we adopt MATLAB function fmincon, and the improved pattern search algorithm in [26] to search for the optimum solution respectively. The results are shown in Table 7 .

Table 6 Modified elements for Situation $5(\delta=0.5)$ (Elements in the directed circuit are in bold)

\begin{tabular}{|c|c|c|c|c|c|c|c|}
\hline Element & $\begin{array}{c}\text { Original } \\
\text { value }\end{array}$ & $\begin{array}{c}\text { Modification } \\
\text { bound }\end{array}$ & $\begin{array}{l}\text { Modified } \\
\text { value }\end{array}$ & Element & $\begin{array}{c}\text { Original } \\
\text { value }\end{array}$ & $\begin{array}{c}\text { Modification } \\
\text { bound }\end{array}$ & $\begin{array}{c}\text { Modified } \\
\text { value }\end{array}$ \\
\hline$a_{12}$ & 7 & {$[6.5,7.5]$} & 6.5 & $a_{34}$ & 0.2 & {$[1 / 9,9]$} & 0.8384 \\
\hline$a_{13}$ & 3 & {$[2.5,3.5]$} & 3.5 & $a_{35}$ & 0.3333 & {$[1 / 9,9]$} & 2.7359 \\
\hline$a_{14}$ & 5 & {$[4.5,5.5]$} & 4.5 & $a_{36}$ & 3 & {$[2.5,3.5]$} & 2.6794 \\
\hline$a_{15}$ & 9 & {$[8.5,9]$} & 9 & $a_{37}$ & 3 & {$[1 / 9,9]$} & 2.5 \\
\hline$a_{16}$ & 3 & {$[2.5,3.5]$} & 3.5 & $a_{45}$ & 9 & {$[8.5,9]$} & 8.5 \\
\hline$a_{17}$ & 5 & {$[4.5,5.5]$} & 5.3787 & $a_{46}$ & 3 & {$[2.5,3.5]$} & 3.1692 \\
\hline$a_{23}$ & 3 & {$[2.5,3.5]$} & 2.5 & $a_{47}$ & 0.3333 & {$[1 / 9,9]$} & 1.8371 \\
\hline$a_{24}$ & 3 & {$[2.5,3.5]$} & 2.5 & $a_{56}$ & 3 & {$[2.5,3.5]$} & 2.5 \\
\hline$a_{25}$ & 5 & {$[4.5,5.5]$} & 4.5 & $a_{57}$ & 0.2 & {$[1 / 9,9]$} & 0.5778 \\
\hline$a_{26}$ & 3 & {$[2.5,3.5]$} & 3.5 & $a_{67}$ & 0.3333 & {$[1 / 3.5,1 / 2.5]$} & 0.4 \\
\hline$a_{27}$ & 3 & {$[2.5,3.5]$} & 2.5 & & & & \\
\hline
\end{tabular}

Table 7 Modified elements by Model 3 and Model 4 in Example 2 (Elements in the directed circuit are in bold)

\begin{tabular}{|c|c|c|c|c|c|c|c|}
\hline \multirow{2}{*}{$\begin{array}{l}\text { Model } \\
\text { being } \\
\text { used }\end{array}$} & \multirow{2}{*}{$\begin{array}{l}\text { Optimization } \\
\text { algorithm }\end{array}$} & \multicolumn{6}{|c|}{ Modified matrix } \\
\hline & & Modified element & $\begin{array}{c}\text { Logical } \\
\text { consistency }\end{array}$ & CR & $\begin{array}{c}\text { Total } \\
\text { perturbation } \varepsilon\end{array}$ & $\begin{array}{l}\text { Maximum } \\
\text { deviation } \sigma\end{array}$ & $\begin{array}{c}\text { Priority vector } \boldsymbol{\omega} \text { and } \\
\text { priority order }\end{array}$ \\
\hline \multirow[b]{2}{*}{ Model 3} & $\begin{array}{l}\text { Improved } \\
\text { pattern search } \\
\text { algorithm }\end{array}$ & $\begin{array}{l}\boldsymbol{X}_{1}=\{6.5,3.1,4.6644,9,3.5 \\
5.0037,2.5017,2.5,4.9697 \\
3.5,2.825, \mathbf{0 . 8 3 8} \mathbf{4}, \mathbf{2 . 7 3 5} \mathbf{9} \\
2.9873, \mathbf{2 . 5}, 8.5,3.0062, \mathbf{1 . 8 3 7} \\
\mathbf{1}, 2.5, \mathbf{0 . 5 7 7} \mathbf{8}, 0.4\}\end{array}$ & Acceptable & 0.1 & 0.2227 & 0.5 & $\begin{array}{c}\{0.4334,0.1773,0.1069,0.1317 \\
0.0418,0.0436,0.0653\} \\
1 \succ 2 \succ 4 \succ 3 \succ 7 \succ 6 \succ 5\end{array}$ \\
\hline & fmincon & $\begin{array}{l}\boldsymbol{X}_{2}=\{6.5147,3.4852,4.514 \\
7,9,3.4852,5.3676,2.515, \\
2.515,4.5147,3.4852,2.515, \\
\mathbf{0 . 8 3 8} \mathbf{4}, \mathbf{2 . 7 3 5} 9,2.689, \mathbf{2 . 5 1 5}, \\
8.514 \quad 5, \quad 3.164 \quad 2, \quad \mathbf{1 . 8 3 7 \quad 1 ,} \\
2.515, \mathbf{0 . 5 7 7} \mathbf{8}, 0.36857\}\end{array}$ & Acceptable & 0.1 & 0.2555 & 0.4855 & $\begin{array}{c}\{0.4373,0.1725,0.1038,0.1336 \\
0.0424,0.0436,0.0669\} \\
1 \succ 2 \succ 4 \succ 3 \succ 7 \succ 6 \succ 5\end{array}$ \\
\hline Model 4 & fmincon & $\begin{array}{l}\boldsymbol{X}_{3}=\{6.5292,3.4709,4.529 \\
2,9,3.4709,5.3787,2.5292, \\
2.5292,4.5292,3.4709,2.529 \\
2, \mathbf{0 . 8 3 8} \mathbf{4}, \mathbf{2 . 7 3 5} \mathbf{9}, 2.6794 \\
\mathbf{2 . 5 2 9} 2,8.5292,3.1692, \mathbf{1 . 8 3 7} \\
\mathbf{1 , 2 . 5 2 9} 2, \mathbf{0 . 5 7 7} \mathbf{8}, 0.3954\}\end{array}$ & Acceptable & 0.1 & 0.2551 & 0.4709 & $\begin{array}{c}\{0.4376,0.1729,0.1037,0.1337 \\
0.0425,0.0439,0.0657\} \\
1 \succ 2 \succ 4 \succ 3 \succ 7 \succ 6 \succ 5\end{array}$ \\
\hline
\end{tabular}

Thus, we should choose $\boldsymbol{X}_{1}$ and $\boldsymbol{X}_{3}$ in Table 7 , since $\varepsilon$ is minimized in $\boldsymbol{X}_{1}$, while $\sigma$ is minimized in $\boldsymbol{X}_{3}$. That is, to minimize the total perturbation, we get $\sigma=0.5$, $\varepsilon=0.2227$; to minimize maximum deviation among all logically consistent elements, we get $\sigma=0.4709$, $\varepsilon=0.2551$.

Comparisons have been made between the proposed method and method in [11] where the HPIBM $\boldsymbol{C}^{0}$ and $\boldsymbol{C}$ are constructed as

$$
\boldsymbol{C}^{0}=\left[\begin{array}{lllllll}
0 & 0 & 0 & 0 & 0 & 0 & 0 \\
0 & 0 & 0 & 0 & 0 & 0 & 0 \\
0 & 0 & 0 & 1 & 1 & 0 & 0 \\
0 & 0 & 0 & 0 & 0 & 0 & 1 \\
0 & 0 & 0 & 0 & 0 & 0 & 1 \\
0 & 0 & 0 & 0 & 0 & 0 & 0 \\
0 & 0 & 2 & 0 & 0 & 0 & 0
\end{array}\right], \quad \boldsymbol{C}=\left[\begin{array}{cccccccc}
1 & 0.4313 & 3.8889 & 1.3886 & 1.9841 & 4.4286 & 1.2705 \\
4.0413 & 1 & 1.8299 & 0.8509 & 1.5796 & 2.3061 & 0.8912 \\
1.0249 & 2.2 & 1 & 9.3598 & 9.9143 & 0.8857 & 0.4667 \\
2.9864 & 2.8476 & 1.1346 & 1 & 0.4095 & 2.4095 & 8.7714 \\
2.9739 & 2.3439 & 0.4074 & 4.6 & 1 & 0.8032 & 8.2804 \\
0.4268 & 1.4571 & 2.3333 & 1.9016 & 4.3333 & 1 & 1.9333 \\
1.9388 & 2.219 & \underline{14.257} & 0.4582 & 1.1879 & 1.5524 & 1
\end{array}\right] .
$$


From $C^{0}$, we can derive the same 3 -node directed circuits as our method: $7 \rightarrow 4 \rightarrow 3 \rightarrow 7,7 \rightarrow 5 \rightarrow 3 \rightarrow 7$. The largest value in matrix $C, 14.257$, corresponds to the non-zero element $c_{73}^{0}=2$ in matrix $\boldsymbol{C}^{0}$. To eliminate the two directed circuits all at once, we just need to redirect $a_{73}$, according to [11],

$$
\tilde{a}_{73}=\frac{7 c_{73}-2}{7-2} a_{73}=6.52 .
$$

By replacing $a_{73}$ and $a_{37}$ with 6.52 and 0.1534 $(=1 / 6.52)$ respectively, the modified PCM is logically con- sistent, but CR is improved from 0.3061 to 0.2401 ( $>0.1)$, see Table 8, which means the modified PCM is still unacceptable in numerical consistency. The other situations of modification are analyzed similarly, and the comparison results are given in Table 8. From Table 8, both our method with $\delta=0$ and method in [11] have failed to attain the acceptable consistent level, but our method with $\delta=0.5$ is feasible (see Table 7), which shows our method is more efficient than that in [11]. Besides, we can see from Table 8 , our method gains better value of CR than method in [11] for each situation.

Table 8 Comparison between our method and method in Example 2 of [11]

\begin{tabular}{|c|c|c|c|c|c|c|c|}
\hline \multirow[b]{2}{*}{ Situation } & \multirow{2}{*}{$\begin{array}{l}\text { Element with } \\
\text { logical error }\end{array}$} & \multicolumn{2}{|c|}{$\begin{array}{l}\text { Modified matrix in our method } \\
\text { (also see Table 5) }\end{array}$} & \multicolumn{4}{|c|}{ Modified matrix by method in [11] } \\
\hline & & $\begin{array}{c}\text { Modified } \\
\text { element }\end{array}$ & CR & $\begin{array}{c}\text { Modified } \\
\text { element }\end{array}$ & CR & $\begin{array}{c}\text { Logical } \\
\text { consistency }\end{array}$ & $\begin{array}{c}\text { Consistent } \\
\text { level }\end{array}$ \\
\hline $\begin{array}{l}\text { Situation } 1 \\
\text { (Assume one } \\
\text { element has } \\
\text { logical error) }\end{array}$ & $a_{37}$ & $a_{37}=0.3588$ & 0.2303 & $\begin{array}{c}a_{73}=6.52 \\
\left(a_{37}=0.1534\right)\end{array}$ & 0.2401 & Acceptable & $\begin{array}{c}\text { Not } \\
\text { acceptable }\end{array}$ \\
\hline \multirow{4}{*}{$\begin{array}{l}\text { Situation } 2 \\
\text { (Assume two } \\
\text { elements have } \\
\text { logical error) }\end{array}$} & $a_{47}, a_{37}$ & $\begin{array}{l}a_{47}=1.2687 \\
a_{37}=0.4688\end{array}$ & 0.206 & $\begin{array}{c}a_{47}=3.96 \\
a_{73}=6.52 \\
\left(a_{37}=0.1534\right)\end{array}$ & 0.2443 & Acceptable & $\begin{array}{c}\text { Not } \\
\text { acceptable }\end{array}$ \\
\hline & $a_{35}, a_{37}$ & $\begin{array}{l}a_{35}=1.7945 \\
a_{37}=0.5047\end{array}$ & 0.1909 & $\begin{array}{c}a_{73}=6.52 \\
\left(a_{37}=0.1534\right) \\
a_{35}=4.4933\end{array}$ & 0.2263 & Acceptable & $\begin{array}{c}\text { Not } \\
\text { acceptable }\end{array}$ \\
\hline & $a_{47}, a_{35}$ & $\begin{array}{l}a_{47}=2.0032 \\
a_{35}=2.4164\end{array}$ & 0.1875 & $\begin{array}{c}a_{47}=3.96 \\
a_{35}=4.4933\end{array}$ & 0.1987 & Acceptable & $\begin{array}{c}\text { Not } \\
\text { acceptable }\end{array}$ \\
\hline & $a_{47}, a_{57}$ & $\begin{array}{l}a_{47}=2.8265 \\
a_{57}=1.0024\end{array}$ & 0.2148 & $\begin{array}{c}a_{47}=3.96 \\
a_{57}=2.2385\end{array}$ & 0.2231 & Acceptable & $\begin{array}{c}\text { Not } \\
\text { acceptable }\end{array}$ \\
\hline \multirow{5}{*}{$\begin{array}{l}\text { Situation } 3 \\
\text { (Assume three } \\
\text { elements have } \\
\text { logical error) }\end{array}$} & $a_{34}, a_{37}, a_{35}$ & $\begin{array}{l}a_{34}=1.0001 \\
a_{37}=0.7879 \\
a_{35}=2.7558\end{array}$ & 0.1608 & $\begin{array}{c}a_{34}=2.5407 \\
a_{73}=6.52 \\
\left(a_{37}=0.1534\right) \\
a_{35}=4.4933\end{array}$ & 0.2205 & Acceptable & $\begin{array}{c}\text { Not } \\
\text { acceptable }\end{array}$ \\
\hline & $a_{47}, a_{37}, a_{35}$ & $\begin{array}{c}a_{47}=1.4608 \\
a_{37}=0.711 \\
a_{35}=1.8671\end{array}$ & 0.1636 & $\begin{array}{c}a_{47}=3.96 \\
a_{73}=6.52 \\
\left(a_{37}=0.1534\right) \\
a_{35}=4.4933\end{array}$ & 0.2333 & Acceptable & $\begin{array}{c}\text { Not } \\
\text { acceptable }\end{array}$ \\
\hline & $a_{47}, a_{34}, a_{35}$ & $\begin{array}{l}a_{47}=1.3968 \\
a_{34}=1.0021 \\
a_{35}=3.4191\end{array}$ & 0.1543 & $\begin{array}{c}a_{47}=3.96 \\
a_{34}=2.5407 \\
a_{35}=4.4933\end{array}$ & 0.1819 & Acceptable & $\begin{array}{c}\text { Not } \\
\text { acceptable }\end{array}$ \\
\hline & $a_{47}, a_{57}, a_{35}$ & $\begin{array}{l}a_{47}=2.5243 \\
a_{57}=1.0001 \\
a_{35}=1.9476\end{array}$ & 0.1717 & $\begin{array}{c}a_{47}=3.96 \\
a_{57}=2.2385 \\
a_{35}=4.4933\end{array}$ & 0.2011 & Acceptable & $\begin{array}{c}\text { Not } \\
\text { acceptable }\end{array}$ \\
\hline & $a_{47}, a_{37}, a_{57}$ & $\begin{array}{l}a_{47}=2.0692 \\
a_{37}=0.7366 \\
a_{57}=1.0001\end{array}$ & 0.1913 & $\begin{array}{c}a_{47}=3.96 \\
a_{73}=6.52 \\
\left(a_{37}=0.1534\right) \\
a_{57}=2.2385\end{array}$ & 0.2562 & Acceptable & $\begin{array}{c}\text { Not } \\
\text { acceptable }\end{array}$ \\
\hline $\begin{array}{l}\text { Situation } 4 \\
\text { (Assume four } \\
\text { elements have } \\
\text { logical error) }\end{array}$ & $a_{34}, a_{47}, a_{35}, a_{57}$ & $\begin{array}{l}a_{34}=1.0001 \\
a_{47}=2.0432 \\
a_{35}=2.5464 \\
a_{57}=1.0001\end{array}$ & 0.1437 & $\begin{array}{c}a_{34}=2.5407 \\
a_{47}=3.96 \\
a_{35}=4.4933 \\
a_{57}=2.2385\end{array}$ & 0.1854 & Acceptable & $\begin{array}{c}\text { Not } \\
\text { acceptable }\end{array}$ \\
\hline
\end{tabular}


Example 3 The $5 \times 5$ PCM $\boldsymbol{A}$, which is also analyzed in [11] and [17], is numerically consistent with $\mathrm{CR}=0.0829<0.1$, but logically inconsistent.

\begin{tabular}{c|ccccc|} 
& 1 & 2 & 3 & 4 & 5 \\
\hline 1 & 1 & $7 / 4$ & $3 / 4$ & $5 / 2$ & $7 / 4$ \\
2 & $4 / 7$ & 1 & $3 / 4$ & $9 / 4$ & $9 / 4$ \\
3 & $4 / 3$ & $4 / 3$ & 1 & $3 / 4$ & $3 / 4$ \\
4 & $2 / 5$ & $4 / 9$ & $4 / 3$ & 1 & $5 / 8$ \\
5 & $4 / 7$ & $4 / 9$ & $4 / 3$ & $8 / 5$ & 1
\end{tabular}

(i) Analyze the logical consistency. By (1) and (4), we have

$$
\boldsymbol{R}=\left[\begin{array}{lllll}
0 & 1 & 0 & 1 & 1 \\
0 & 0 & 0 & 1 & 1 \\
1 & 1 & 0 & 0 & 0 \\
0 & 0 & 1 & 0 & 0 \\
0 & 0 & 1 & 1 & 0
\end{array}\right], \quad \boldsymbol{T}=\left[\begin{array}{lllll}
1 & 1 & 1 & 1 & 1 \\
1 & 1 & 1 & 1 & 1 \\
1 & 1 & 1 & 1 & 1 \\
1 & 1 & 1 & 1 & 1 \\
1 & 1 & 1 & 1 & 1
\end{array}\right]
$$

There exist five elements equal to 1 on the main diagonal of reachable matrix $\boldsymbol{T}, t_{11}=1, t_{22}=1, t_{33}=1$, $t_{44}=1, t_{55}=1$, and $m=5$. Similar to Example 2, we obtain four 3-node directed circuits: $4 \rightarrow 3 \rightarrow 2 \rightarrow 4$, $4 \rightarrow 3 \rightarrow 1 \rightarrow 4,5 \rightarrow 3 \rightarrow 2 \rightarrow 5,5 \rightarrow 3 \rightarrow 1 \rightarrow 5$.

This example is featured with lots of 3-node directed circuits, which has too many possible modification combinations to judge manually. Therefore, we have developed computer programs to handle this task according to the procedure in Section 3.2.2.

(ii) Improve the consistency level.

i) Find all the possible elements in 3-node directed circuits. There are eight such elements, $a_{13}, a_{14}, a_{15}, a_{23}$, $a_{24}, a_{25}, a_{34}, a_{35}$.

ii) List all the possible modification combinations which can eliminate all the directed circuits. In theory, there are
$\mathrm{C}_{8}^{1}+\mathrm{C}_{8}^{2}+\mathrm{C}_{8}^{3}+\cdots+\mathrm{C}_{8}^{7}=254$ modification combinations, however, in many cases, redirect all elements in the combination will not eliminate all the directed circuits. Thus, we have to choose the possible modification combinations by (2) and Corollary 1. For example, the combination $\left\{a_{34}\right.$, $\left.a_{35}\right\}$ is chosen, we firstly redirect $a_{34}$ and $a_{35}$ using (13), which means both $a_{34}$ and $a_{35}$ are changed from $3 / 4$ to certain values greater than 1 . Though we do not know the changed value of $a_{34}$ and $a_{35}$, we can derive the modified preference matrix $\boldsymbol{R}^{\prime}$ using (1), and the number of minimum directed circuits $c$ by $\boldsymbol{R}^{\prime}$ using (2).

$$
\boldsymbol{R}^{\prime}=\left[\begin{array}{lllll}
0 & 1 & 0 & 1 & 1 \\
0 & 0 & 0 & 1 & 1 \\
1 & 1 & 0 & 1 & 1 \\
0 & 0 & 0 & 0 & 0 \\
0 & 0 & 0 & 1 & 0
\end{array}\right]
$$

Since $c=1 / 6 \times 5 \times 4 \times 3-1 / 2 \times[3 \times(3-1)+2 \times(2-$ $1)+4 \times(4-1)+0 \times(0-1)+1 \times(1-1)]=0$, we can conclude that there is no directed circuits with redirections of $a_{34}$ and $a_{35}$, and the modified PCM will be logically consistent according to Corollary 1. Similarly, the other possible modification combinations are analyzed by computer programs considering the large combination number (254 modification possibilities), and we have chosen 30 different feasible combinations that can eliminate all directed circuits (realize $c=0$ ) as shown partially in Table 9.

Take the combination $\left\{a_{34}, a_{35}\right\}$ as an example, see Table 9 . By modifying $a_{34}$ from $3 / 4$ to $3.4593, a_{35}$ from $3 / 4$ to 2.4081 , the optimized PCM by Model 1 is logically consistent, and numerically consistent with $\mathrm{CR}=0.0135$, which is smaller than Kou et al. [11]'s CR=0.016 $4\left(a_{34}=\right.$ $\left.2.5111, a_{35}=1.934\right)$ and Siraj et al. [17]'s CR=0.055 $\left(a_{34}=0.99, a_{35}=0.99\right)$.

Table 9 Feasible output results for different situations in Example 3

\begin{tabular}{cccc}
\hline Situation & Element with logical error & Modified element & CR \\
\hline Situation 1 (Assume two & $a_{35}, a_{34}$ & $a_{34}=3.4593, a_{35}=2.4081$ & 0.0135 \\
elements have logical error) & $a_{13}, a_{23}$ & $a_{13}=3.306, a_{23}=2.523$ & 0.0143 \\
\hline & $a_{34}, a_{23}, a_{13}$ & $a_{34}=1.0395, a_{23}=2.1531, a_{13}=2.8033$ & 0.0124 \\
& $a_{34}, a_{23}, a_{35}$ & $a_{34}=2.9758, a_{13}=1.0001, a_{35}=2.1088$ & 0.0124 \\
& $a_{34}, a_{13}, a_{25}$ & $a_{34}=1.7328, a_{13}=1.6641, a_{25}=0.9187$ & 0.0115 \\
Situation 2 (Assume three & $a_{34}, a_{35}, a_{25}$ & $a_{34}=3.0736, a_{35}=1.8171, a_{25}=0.9999$ & 0.0073 \\
elements have logical error) & $a_{34}, a_{25}, a_{15}$ & $a_{34}=2.2853, a_{25}=0.76852, a_{15}=0.9999$ & 0.0201 \\
& $a_{24}, a_{13}, a_{25}$ & $a_{24}=0.9628, a_{13}=2.1643, a_{25}=0.6971$ & 0.0119 \\
& $a_{23}, a_{13}, a_{25}$ & $a_{23}=1.8953, a_{13}=2.9543, a_{25}=0.9999$ & 0.0087 \\
\hline \multirow{2}{*}{ Other situations (Assume four } & $\ldots$ & & $\ldots$ \\
or more elements have logical & $a_{34}, a_{24}, a_{13}, a_{35}, a_{25}$ & $a_{34}=1.5449, a_{24}=0.9999, a_{13}=1.5325$, & 0.0028 \\
error) & $\ldots$ & $a_{35}=1.0617, a_{25}=0.7982$ & $\ldots$ \\
\hline
\end{tabular}


Among these 30 feasible combinations in Table 9, the combination $\left\{a_{34}, a_{24}, a_{13}, a_{35}, a_{25}\right\}$ has minimum CR (= 0.002809$)$, and the combinations $\left\{a_{34}, a_{35}\right\}$ and $\left\{a_{13}\right.$, $\left.a_{23}\right\}$ have minimum modification elements, i.e., two elements. Theoretically, all the 30 combinations can derive feasible PCM with the acceptable consistent level by Definition 7. Thus the problem is which combination is the best choice. In general, we can deal with it by the following principles. If the DM is asked to choose which elements or combinations to redirect (or elements with logical error), we can attain the modified PCM according to the chosen combinations. If no DM is involved, to make the problem easier and the modification smaller, we can choose the combinations with minimum modification elements, e.g., $\left\{a_{34}, a_{35}\right\}$ and $\left\{a_{13}, a_{23}\right\}$ will be the optimum combinations in Example 3, which is the same as the results in [11], but easier and more comprehensive in that we list all feasible results and just choose the results with minimum modification elements, instead of identifying and analyzing which combinations need to be modified as [11]. Owning to these advantages, our method can be realized by computer programs automatically to list all the feasible output results as Table 9.

Example 4 The $8 \times 8$ PCM $\boldsymbol{A}$, which is also analyzed in [11] and [29], is inconsistent with $\mathrm{CR}=0.1047>0.1$.

\begin{tabular}{c|cccccccc|} 
& 1 & 2 & 3 & 4 & 5 & 6 & 7 & 8 \\
\hline 1 & 1 & 2 & $1 / 2$ & 2 & $1 / 2$ & 2 & $1 / 2$ & 2 \\
2 & $1 / 2$ & 1 & 4 & 1 & $1 / 4$ & 1 & $1 / 4$ & 1 \\
3 & 2 & $1 / 4$ & 1 & 4 & 1 & 4 & 1 & 4 \\
4 & $1 / 2$ & 1 & $1 / 4$ & 1 & $1 / 4$ & 1 & $1 / 4$ & 1 \\
5 & 2 & 4 & 1 & 4 & 1 & 4 & 1 & 4 \\
6 & $1 / 2$ & 1 & $1 / 4$ & 1 & $1 / 4$ & 1 & $1 / 4$ & 1 \\
7 & 2 & 4 & 1 & 4 & 1 & 4 & 1 & 4 \\
8 & $1 / 2$ & 1 & $1 / 4$ & 1 & $1 / 4$ & 1 & $1 / 4$ & 1
\end{tabular}

According to Assumption 1 and (3), the PCM $\boldsymbol{A}$ is transformed to an equivalent NOE-PCM $\boldsymbol{A}^{\prime}$.

Since nine elements are equal to 1 in the upper diagonal triangular matrix of $\boldsymbol{A}$, there should be $2^{9}=512$ different cases of the modified NOE-PCMs, where 12 cases have minimum $c(=1)$, i.e., only one 3-node directed circuit inside these NOE-PCMs. We randomly choose a modified NOE-PCM $\boldsymbol{A}^{\prime}$ with $c=1$.

$$
\boldsymbol{A}^{\prime}=\left[\begin{array}{cccccccc}
1 & 2 & 1 / 2 & 2 & 1 / 2 & 2 & 1 / 2 & 2 \\
1 / 2 & 1 & 4 & 1.0001 & 1 / 4 & 1.0001 & 1 / 4 & 1.0001 \\
2 & 1 / 4 & 1 & 4 & 0.9999 & 4 & 0.9999 & 4 \\
1 / 2 & 0.9999 & 1 / 4 & 1 & 1 / 4 & 0.9999 & 1 / 4 & 0.9999 \\
2 & 4 & 1.0001 & 4 & 1 & 4 & 0.9999 & 4 \\
1 / 2 & 0.9999 & 1 / 4 & 1.0001 & 1 / 4 & 1 & 1 / 4 & 0.9999 \\
2 & 4 & 1.0001 & 4 & 1.0001 & 4 & 1 & 4 \\
1 / 2 & 0.9999 & 1 / 4 & 1.0001 & 1 / 4 & 1.0001 & 1 / 4 & 1
\end{array}\right]
$$

(i) Analyze the logical consistency of $\boldsymbol{A}^{\prime}$. By (1) and (4), we have

$$
\begin{aligned}
\boldsymbol{R} & =\left[\begin{array}{llllllll}
0 & 1 & 0 & 1 & 0 & 1 & 0 & 1 \\
0 & 0 & 1 & 1 & 0 & 1 & 0 & 1 \\
1 & 0 & 0 & 1 & 0 & 1 & 0 & 1 \\
0 & 0 & 0 & 0 & 0 & 0 & 0 & 0 \\
1 & 1 & 1 & 1 & 0 & 1 & 0 & 1 \\
0 & 0 & 0 & 1 & 0 & 0 & 0 & 0 \\
1 & 1 & 1 & 1 & 1 & 1 & 0 & 1 \\
0 & 0 & 0 & 1 & 0 & 1 & 0 & 0
\end{array}\right], \\
\boldsymbol{T} & =\left[\begin{array}{llllllll}
1 & 1 & 1 & 1 & 0 & 1 & 0 & 1 \\
1 & 1 & 1 & 1 & 0 & 1 & 0 & 1 \\
1 & 1 & 1 & 1 & 0 & 1 & 0 & 1 \\
0 & 0 & 0 & 0 & 0 & 0 & 0 & 0 \\
1 & 1 & 1 & 1 & 0 & 1 & 0 & 1 \\
0 & 0 & 0 & 1 & 0 & 0 & 0 & 0 \\
1 & 1 & 1 & 1 & 1 & 1 & 0 & 1 \\
0 & 0 & 0 & 1 & 0 & 1 & 0 & 0
\end{array}\right] .
\end{aligned}
$$

There exist three elements equal to 1 on the main dia- gonal of reachable matrix $\boldsymbol{T}, t_{11}=1, t_{22}=1, t_{33}=1$ and $m=3$. By using the 3 -node directed circuit method, we obtain one 3 -node directed circuit: $3 \rightarrow 1 \rightarrow 2 \rightarrow 3$.

(ii) Improve the consistency level. We list all the possible modification combinations as follows.

Situation 1 Suppose there is one element that has logical error in the 3-node directed circuit, then we have the following possible modification combinations, $\left\{a_{23}\right\}$, $\left\{a_{13}\right\},\left\{a_{12}\right\}$. By using Model 1 , the results are listed in Table 10 . We can see that it is better to modify $a_{23}$ because the modified PCM is not only logically consistent, but also numerically perfect consistent with $\mathrm{CR}=0$.

Other situations Since Situation 1 has reached acceptable consistent level by just modifying one element with logical error $\left(a_{23}\right)$ and $\varepsilon=0$ and $\sigma=0$ by (15) and (16), as a result, we will not consider other situations, because it is unnecessary to modify other elements which can only help to increase the number of modified elements, and to 
make the two indices, $\varepsilon$ and $\sigma$, greater than 0 . In other words, the result for Situation 1 is good enough, and the other situations will not be considered. Note that our re- sults are the same as those found in [11] and [29], which proves the efficiency and effectiveness of our method when dealing with PCM with lots of elements equal to 1.

Table 10 Output results for Situation 1 in Example 4

\begin{tabular}{|c|c|c|c|c|c|c|}
\hline \multirow[b]{2}{*}{ Situation } & \multirow{2}{*}{$\begin{array}{l}\text { Element with } \\
\text { logical error }\end{array}$} & \multicolumn{5}{|c|}{ Modified matrix } \\
\hline & & $\begin{array}{c}\text { Modified } \\
\text { element }\end{array}$ & $\begin{array}{c}\text { Logical } \\
\text { consistency }\end{array}$ & CR & $\begin{array}{c}\text { Consistent } \\
\text { level }\end{array}$ & $\begin{array}{l}\text { Priority vector } \boldsymbol{\omega} \text { and } \\
\text { priority order }\end{array}$ \\
\hline \multirow{3}{*}{$\begin{array}{l}\text { Situation } 1 \\
\text { (Assume one } \\
\text { element has } \\
\text { logical error) }\end{array}$} & $\begin{array}{l}a_{23} \text { redirect, and other ele- } \\
\text { ments keep unchanged }\end{array}$ & $a_{23}=0.25$ & Acceptable & 0 & Acceptable & $\begin{array}{l}\{0.1111,0.0556,0.2222,0.0556, \\
0.2222,0.0556,0.2222,0.0556\} \\
7 \sim 5 \sim 3 \succ 1 \succ 8 \sim 6 \sim 4 \sim 2\end{array}$ \\
\hline & $\begin{array}{l}a_{13} \text { redirect, and other ele- } \\
\text { ments keep unchanged }\end{array}$ & $a_{13}=1.0005$ & Acceptable & 0.0992 & Acceptable & $\begin{array}{c}\{0.1187,0.1203,0.1564,0.055 \\
0.2199,0.055,0.2199,0.055\} \\
7 \sim 5 \succ 3 \succ 2 \succ 1 \succ 8 \sim 6 \sim 4\end{array}$ \\
\hline & $\begin{array}{l}a_{12} \text { redirect, and other ele- } \\
\text { ments keep unchanged }\end{array}$ & $a_{12}=0.9996$ & Acceptable & 0.0992 & Acceptable & $\begin{array}{l}\{0.0959,0.1301,0.1665,0.0552 \\
0.2209,0.0552,0.2209,0.0552\} \\
7 \sim 5 \succ 3 \succ 2 \succ 1 \succ 8 \sim 6 \sim 4\end{array}$ \\
\hline
\end{tabular}

\section{Conclusions}

This paper proposes an approach to derive an acceptable PCM from an inconsistent one based on the concept of the acceptable consistent level, which includes acceptable logical and numerical consistency.

It is suggested that logical inconsistency should be improved firstly, for that purpose, we propose two logical consistency identification methods: reachable matrix method and 3-node directed circuit method. Then, four optimization models are constructed, and the consistency improving strategy is designed to realize the acceptable consistent level. Illustrative examples show the effectiveness and feasibility of our method, and comparison studies are made among our method and previous studies, where we find that the logical error still exists in the improved matrix in previous studies by [5] and [6] (see Example 1), and that our method is more efficient than that in [11] because the latter may fail to obtain an acceptable modification (see Example 2), and our method is easier to realize by computer programming and more comprehensive (see Example 3) than that in [11], [17], etc. Meanwhile, it can tackle NOE-PCM effectively (see Example 4). It must be emphasized that such an approach should only be used as a decision aid, which the DM uses as a reference to help modify the original PCM. Thus, we design procedures with DM's involvement to fully consider the DM's modification advices, as well as without DM's involvement if the DM is not available or the DM is confused with complicated logical errors.

The main contributions of this paper are as follows:

(i) The reachable matrix method is proposed to identify all 3-node directed circuits, and its theoretical basis is proved.

(ii) The 3-node directed circuit method from graph the- ory is adopted to identify logical inconsistency, which is convenient to realize by computer programming and more efficient than existing directed circuits detecting methods that are used manually.

(iii) We propose to modify elements differently according to whether it is in a 3-node directed circuit, i.e., with logical error: the elements with logical error are allowed to be modified within a larger modification range so that the logical error can be eliminated firstly, while the elements without logical error are only allowed to be modified within an acceptable modification bound so that the linguistic meaning is not changed. And we propose that the total modification of PCM should not consider the elements modified by logical inconsistency because they are wrong judgments in essence.

(iv) We propose four optimization models, which can cover different modification situations and ensure the improved PCM optimal. Besides, the method of transforming PCM with many elements equal to 1 is proposed and proved to be applicable.

A future research issue is on whether our methods would be extended to improve the comparison matrix with other different entries, such as POM entries [12,13], as well as group decision-making problems [30].

\section{References}

[1] HO W. Integrated analytic hierarchy process and its applications: a literature review. European Journal of Operational Research, 2008, 186(1): $211-228$.

[2] VAIDYA O S, KUMAR S. Analytic hierarchy process: an overview of applications. European Journal of Operational Research, 2006, 169(1): 1-29.

[3] STAN L, CONKLIN W M. Robust estimation of priorities in the AHP. European Journal of Operational Research, 2002, 137(1): $110-122$.

[4] TSENG T Y, LIN S W, HUANG C L, et al. Inconsistency adjustment in the AHP using the complete transitivity convergence algorithm. Proc. of the IEEE International Conference 
on Systems, Man and Cybernetics, 2006: $2808-2812$.

[5] XU Z S, WEI C P. A consistency improving method in the analytic hierarchy process. European Journal of Operational Research, 1999, 116(2): $443-449$.

[6] CAO D, LEUNG L C, LAW J S. Modifying inconsistent comparison matrix in analytic hierarchy process: a heuristic approach. Decision Support Systems, 2008, 44(4): 944-953.

[7] ERGU D, KOU G, PENG Y, et al. A simple method to improve the consistency ratio of the pair-wise comparison matrix in ANP. European Journal of Operational Research, 2011, 213(1): $246-259$.

[8] PEREIRA V, COSTA H G. Nonlinear programming applied to the reduction of inconsistency in the AHP method. Annals of Operations Research, 2015, 229(1): 635-655.

[9] XU Y, WANG H. An eigenvector method based consistency improving procedure for fuzzy and multiplicative preference relations. Journal of Intelligent \& Fuzzy Systems, 2017, 33(3): $1491-1503$.

[10] ASHKAN N. Optimizing consistency improvement of positive reciprocal matrices with implications for Monte Carlo analytic hierarchy process. Computers \& Industrial Engineering, 2018, 124: $113-124$.

[11] KOU G, ERGU D, SHANG J. Enhancing data consistency in decision matrix: adapting Hadamard model to mitigate judgment contradiction. European Journal of Operational Research, 2014, 236(1): 261-271.

[12] YUEN K K F. Pairwise opposite matrix and its cognitive prioritization operators: comparisons with pairwise reciprocal matrix and analytic prioritization operators. Journal of the Operational Research Society, 2012, 63(3): 322-338.

[13] YUEN K K F. The primitive cognitive network process in healthcare and medical decision making: comparisons with the analytic hierarchy process. Applied Soft Computing, 2014, 14: $109-119$.

[14] FAN Z P, XIAO S H. The consistency and ranking method for comparison matrix with linguistic assessment. Systems Engineering - Theory \& Practice, 2002, 22(5): 87-91. (in Chinese)

[15] GASS S I. Tournaments, transitivity and pairwise comparison matrices. Journal of the Operational Research Society, 1998, 49(6): $616-624$.

[16] DU D F. Graph theory in operations research. Beijing: Beihang University Press, 1990. (in Chinese)

[17] SIRAJ S, MIKHAILOV L. A heuristic method to rectify intransitive judgments in pairwise comparison matrices. European Journal of Operational Research, 2012, 216 (2): $420-$ 428.

[18] XU Z S. Incomplete complementary judgment matrix. Systems Engineering — Theory \& Practice, 2004, 24(6): 91 - 97. (in Chinese)

[19] WU S H, XIE J, LIU X D, et al. A marginal optimization method to improve the inconsistent comparison matrix in the analytic hierarchy process. Journal of Systems Engineering and Electronics, 2017, 28(6): $1141-1151$.

[20] KOU G, LIN C S. A cosine maximization method for the priority vector derivation in AHP. European Journal of Operational Research, 2014, 235(1): 225-232.

[21] SAATY T L. A scaling method for priorities in hierarchical structures. Journal of Mathematical Psychology, 1977, 15: $234-281$.

[22] CHU A T W, KALABA R E, SPINGARN K. A comparison of two methods for determining the weights of belonging to fuzzy sets. Journal of Optimization Theory and Applications, 1979, 27(4): $531-538$.

[23] SRDJEVIC B. Combining different prioritization methods in the analytic hierarchy process synthesis. Computer and Operation Research, 2005, 32(7): $1897-1919$.
[24] CRAWFORD G, WILLIAMS C. A note on the analysis of subjective judgment matrices. Journal of Mathematical Psychology, 1985, 29(4): 387-405.

[25] WANG Y Y, CHEN P, SU Y. A new algorithm to find all elementary circuits of a directed graph. Journal of Shaanxi Normal University (Natural Science Edition), 2008, 36(4): $12-$ 15. (in Chinese)

[26] WU S H, LIU X D, JIA Y L, et al. New optimization method to improve the inconsistent comparison matrix in the analytic hierarchy process. Control and Decision, 2016, 33(11): $2106-$ 2112. (in Chinese)

[27] SAATY T L. Decision-making with the AHP: why is the principal eigenvector necessary. European Journal of Operational Research, 2003, 145(1): 85-91.

[28] BENÍTEZ J, DELGADO-GALVÁN X, IZQUIERDO J, et al. Improving consistency in AHP decision-making processes. Applied Mathematics and Computation, 2012, 219(5): 2432 2441.

[29] KWIESIELEWICZ M, VAN UDEN E. Inconsistent and contradictory judgements in pairwise comparison method in the AHP. Computer and Operation Research, 2004, 31(5): $713-$ 719.

[30] XU Y, WEN X, ZHANG W. A two-stage consensus method for large-scale multi-attribute group decision making with an application to earthquake shelter selection. Computers \& Industrial Engineering, 2018, 116: 113-129.

\section{Biographies}

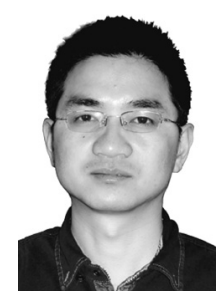

WU Shihui was born in 1982. He received his M.S. and $\mathrm{Ph} . \mathrm{D}$. degrees in management science and engineering from Air Force Engineering University, Xi' an, China, in 2007 and 2010, respectively. He is currently a lecturer in Air Force Engineering University. His research interests focus on decision theory, simulation optimization and so on.

E-mail:wu_s_h82@sina.com

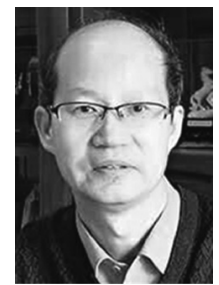

LIU Xiaodong was born in 1966. He is currently a professor and Ph.D. student supervisor in Air Force Engineering University. His research interests focus on management of weapon equipment, weapon equipment economics and so on.

E-mail: liuxiaodong@163.com

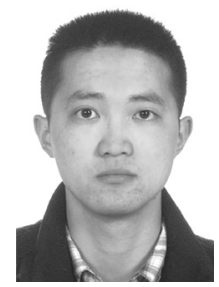

LI Zhengxin was born in 1982 . He is currently a $\mathrm{Ph} . D$. candidate and a lecturer in Air Force Engineering University. His research interests focus on data mining, machine learning, time series analysis and so on.

E-mail: lizhengxin_2005@163.com

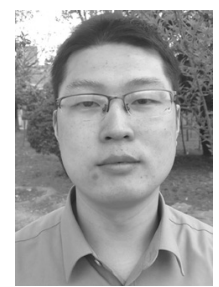

ZHOU Yu was born in 1983. He is currently a Ph.D. candidate and a lecturer in Air Force Engineering University. His research interests focus on test and evaluation for military equipment and so on. E-mail: zhouyu_gfkd@126.com 